\title{
Joint power allocation for MIMO-OFDM full-duplex relaying communications
}

\author{
Hoang D. Tuan ${ }^{1}$, Duy T. Ngo ${ }^{*}$ (D) and Ho H. M. Tam ${ }^{1}$
}

\begin{abstract}
In this paper, we address the problem of joint power allocation in a two-hop MIMO-OFDM network, where two full-duplex users communicate with each other via an amplify-and-forward relay. We consider a general model in which the full-duplex relay can forward the received message in either one-way or two-way mode. Our aim is to maximize the instantaneous end-to-end total throughput, subject to (i) the separate sum-power constraints at individual nodes or (ii) the joint sum-power constraint of the whole network. The formulated problems are large-scale nonconvex optimization problems, for which efficient and optimal solutions are currently not available. Using the successive convex approximation approach, we develop novel iterative algorithms of extremely low complexity which are especially suitable for large-scale computation. In each iteration, a simple closed-form solution is derived for the approximated convex program. The proposed algorithms guarantee to converge to at least a local optimum of the nonconvex problems. Numerical results verify that the devised solutions converge quickly, and that our optimal power allocation schemes significantly improve the throughput of MIMO-OFDM full-duplex one-way/two-way relaying over the conventional half-duplex relaying strategy.
\end{abstract}

Keywords: Iterative optimization, MIMO, OFDM, Power allocation, Successive convex programming

\section{Introduction}

The fifth-generation (5G) wireless networks target a 1,000 -fold increase in the network capacity to meet the ever growing user demands for high-speed and ubiquitous network access. To support such an ambitious goal, multiple-input multiple-output (MIMO) communications and cooperative orthogonal frequency division multiplexing (OFDM) relaying techniques play a key role in enhancing spectral efficiency and improving link reliability. MIMO transmission and reception increase the channel capacity through spatial multiplexing, modulation and coding. Cooperative OFDM relaying provides greater coverage areas without having to deploy costly additional base stations.

OFDM relays are traditionally designed for the halfduplexing (HD) mode, where signal transmission and reception take place in different time slots or frequency bands [1]. Only after fully receiving a data packet, the HD relay nodes forward it to the destination. Full-duplexing

\footnotetext{
*Correspondence: duy.ngo@newcastle.edu.au

${ }^{2}$ School of Electrical Engineering and Computing, The University of Newcastle,

University Drive, Callaghan, NSW 2308, Australia

Full list of author information is available at the end of the article
}

(FD) has recently been proposed as one of the key $5 \mathrm{G}$ transceiver techniques with the hope of doubling the spectral efficiency $[2,3]$. With simultaneous signal transmission and reception in the same time slot and on the same frequency band, an FD relay node transmits a packet while receiving another packet, thereby significantly reducing the end-to-end delay. Such bidirectional communication on the same radio resource block was assumed technically impossible, due to the huge self-interference (SI) introduced by the transmit antenna to the receive antenna on the same device. Only recently, advances in hardware design have suppressed the SI to a level potentially suitable for practical FD applications [4-6].

Two-way relaying strategies [7] have attracted considerable research attention, due to their potential to provide substantially higher spectral efficiency than the conventional one-way relaying counterpart [8-15]. Under the FD two-way relaying, the overall network throughput could further be enhanced by exchanging data in only one time slot as opposed to four in the HD one-way relaying. However, the tradeoff is that an FD two-way relaying scheme suffers even more severe interferences than the FD oneway relaying. It is the direct result of allowing concurrent 
transmissions at both communication ends. Importantly, the rise in the interference level may null out any throughput gain achieved by using fewer time slots.

Efficient power allocation to realize the potential gains of the MIMO-OFDM FD two-way relaying strategy remains an open research topic. Such an allocation is still underdeveloped even for the conventional MIMO-OFDM HD one-way relaying networks. In [16], the problem of power allocation for amplify-and-forward (AF) HD oneway relays is investigated with the aim of maximizing the instantaneous sum throughput. Since the objective function is not jointly but separately concave in the source and relay power variables, [16] proposes alternating optimization at the source and at the relay with individual per-node transmit power constraints. For the joint sumpower constraint at both the source and the relay, [16] resorts to a high signal-to-noise ratio (SNR) approximation for the throughput to become a jointly concave function in the source and relay power variables ([17], Prop. 1 and Appendix B). Although a closed-form optimal solution is available for the convex reformulation, such an approximation at high SNR regions does not always hold in practice. OFDM subchannels tend to be assigned with very different transmit power levels. Good subchannels are typically allocated more power to achieve high SNRs while the bad subchannels may even get zero SNRs. With the high SNR approximation of [16], the original nonconvex program is transformed to an inequivalent optimization problem. Indeed, $[16,17]$ provide upper bound maximization for the original nonconvex maximization, where lower bound maximization should always be preferred. Moreover, the solutions found by either alternating optimization or convex relaxation, in general, may not even satisfy the Karush-Kuhn-Tucker (KKT) necessary conditions for optimality.

The power allocation in a MIMO OFDM two-hop network with FD one-way/two-way relaying is an even more challenging problem. To the best of our knowledge, there currently exists no efficient computational solution that guarantees optimality for this problem. It is unlikely that such a highly nonconvex problem can be solved via just one relaxed convex optimization as in [16, 17]. In this paper, we develop new iterative algorithms of extremely low complexity to jointly allocate the transmit power at the sources and the relay. The key contributions of our work are summarized as follows.

- Novel algorithmic development. We address the nonconvex optimization problem formulations via solving a sequence of convex programs in the complete set of source and relay power variables. The proposed approach applies equally well to both separate and joint sum-power constraints. Our convex approximations are far from trivial even in the simplest scenario of MIMO-OFDM HD one-way relaying considered in [16]. With a new bounding technique, the devised algorithms are novel even from an optimization-theoretic perspective.

- Low-complexity locally optimal algorithms for largescale computation. As each iteration of the algorithms yields an improved solution, they always guarantee to converge to at least a local optimum of the original nonconvex problems. Importantly, unlike $[18,19]$ we derive simple closed-form suboptimal solutions for the convex program in each iteration, which require extremely low computational complexity. Numerical experiments demonstrate that the number of iterations required for our algorithms to converge is small. These two features make our algorithms particularly suitable for large-scale computation.

- Throughput performance improvement. The efficient power allocation schemes markedly enhance the throughput of the MIMO-OFDM FD one-way/two-way relaying over the HD relaying strategies.

The rest of this paper is organized as follows. Section 2 presents the nonconvex problem formulations for power allocation in a MIMO-OFDM relaying network. Sections 3 and 4 propose the optimal power allocation algorithms for two-way and one-way relaying cases, respectively. Section 5 verifies the performance of our proposed algorithms via numerical examples. Finally, Section 6 concludes the paper.

Notation. Boldfaced symbols are used for optimization variables whereas non-boldfaced symbols are for deterministic terms, regardless of whether they are matrix, vector or scalar. The dimensions of these symbols are interpreted from context, and they will be explicitly specified if there is any ambiguity.

\section{System Model and Problem Formulations 2.1 MIMO-OFDM FD Two-Way Relaying}

Consider a two-way relaying network in Fig. 1. Two user terminals 1 and 2 (each equipped with $N$ antennas) exchange information with the help of a relay (also equipped with $N$ antennas) using FD transmissions over $K$ OFDM subcarriers. Denote the index set of the OFDM subcarriers as $\mathcal{K} \triangleq\{1, \ldots, K\}$. We assume there is no direct link between the two users, e.g., building structures prevent signal penetration. The channel impulse response is assumed to be time-invariant during the time for exchanging information. Furthermore, full channel state information is made available at the nodes by some high-performing channel estimation mechanism in place. A central processing unit is employed to collect all the channel state information from the nodes (via either wireline or wireless links), perform the network optimization 


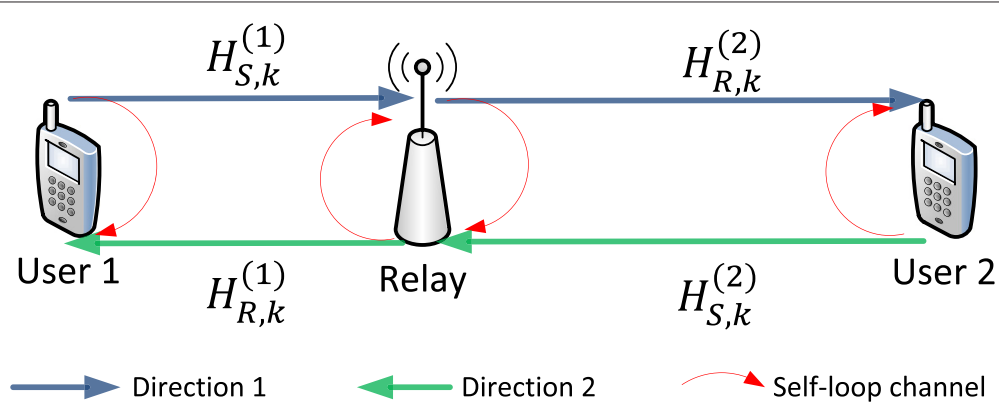

Fig. 1 Two-way MIMO-OFDM relaying with two FD users and one FD relay

and disseminate the computational solution back to the nodes.

Data symbol $s_{k}^{(\ell)} \in \mathbb{C}^{N}$ from user $\ell \in\{1,2\}$ is linearly precoded before transmitting on subcarrier $k \in \mathcal{K}$ as $\tilde{s}_{k}^{(\ell)}=\Psi_{k}^{(\ell)} s_{k}^{(\ell)}$, where $\Psi_{k}^{(\ell)} \in \mathbb{C}^{N \times N}$ is the transmit precoding matrix on subcarrier $k$ by user $\ell$. In the multiple access (MA) phase, the received vector symbol on subcarrier $k$ at the relay is

$$
y_{R, k}=H_{S, k}^{(1)} \tilde{s}_{k}^{(1)}+H_{S, k}^{(2)} \tilde{s}_{k}^{(2)}+e_{\mathrm{Ll}, k}+\tilde{w}_{R, k},
$$

where $H_{S, k}^{(\ell)} \in \mathbb{C}^{N \times N}$ is the MIMO channel matrix between user $\ell$ and the relay on subcarrier $k ; e_{\mathrm{LI}, k} \in \mathbb{C}^{N}$ is the relay FD loop interference on subcarrier $k$; and $\tilde{w}_{R, k} \in$ $\mathbb{C}^{N}$ is the additive zero-mean Gaussian noise with covariance $\mathcal{R}_{R}$ encompassing all OFDM impairments such as intercarrier power leakage, narrow band interferences, channel estimation error and baseband noise [20-22]. The reader is referred to [23-25] and references therein for the suppression techniques of these impairments.

In the broadcast $(\mathrm{BC})$ phase, the relay multiplies the received signal vector on subcarrier $k$ by a matrix $F_{k}$ and broadcasts the processed signal vector to both users 1 and 2. The received signal vector at user $\ell \in\{1,2\}$ is expressed as:

$$
\begin{aligned}
\tilde{y}_{D, k}^{(\ell)}= & H_{R, k}^{(\ell)} F_{k}\left(H_{S, k}^{(1)} \tilde{s}_{k}^{(1)}+H_{S, k}^{(2)} \tilde{s}_{k}^{(2)}+e_{\mathrm{Ll}, k}+\tilde{w}_{R, k}\right)+e_{\mathrm{Ll}, k}^{(\ell)}+\tilde{w}_{D, k}^{(\ell)} \\
= & \underbrace{H_{R, k}^{(\ell)} F_{k} H_{S, k}^{(3-\ell)} \Psi^{(3-\ell)} s_{k}^{(3-\ell)}}_{\text {desired signal }}+\underbrace{H_{R, k}^{(\ell)} F_{k} H_{S, k}^{(\ell)} \Psi^{(\ell)} s_{k}^{(\ell)}}_{\text {back-propagating self-interference }} \\
& +\underbrace{H_{R, k}^{(\ell)} F_{k} e_{\mathrm{Ll}, k}}_{\text {amplified relay loop interference }}+\underbrace{H_{R, k}^{(\ell)} F_{k} \tilde{w}_{R, k}}_{\text {amplified noise }} \\
& +\underbrace{e_{\mathrm{Ll}, k}^{(\ell)}}_{\text {terminal's loop interference }}+\tilde{w}_{D, k}^{(\ell)},
\end{aligned}
$$

where $H_{R, k}^{(\ell)} \in \mathbb{C}^{N \times N}$ is the MIMO channel matrix between the relay and user $\ell$ on subcarrier $k ; e_{\mathrm{LI}, k}^{(\ell)} \in \mathbb{C}^{N}$ is the self-loop interference at user $\ell$ due to FD transmissions; and $\tilde{w}_{D, k}^{(\ell)}$ is the zero-mean Gaussian noise at user $\ell$ with covariance $\mathcal{R}_{D}^{(\ell)}$ encompassing all impairments such as intercarrier power leakage, narrow band interferences, channel estimation error and baseband noise [20-22].

Without loss of generality, we assume that $H_{S, k}^{(\ell)}$ and $H_{R, k}^{(\ell)}$ are nonsingular for both $\ell \in\{1,2\}$. Accordingly, they can be represented by the singular value decomposition (SVD) as:

$$
\begin{aligned}
{\left[H_{S, k}^{(1)} H_{S, k}^{(2)}\right.} & =V_{S, k} \Lambda_{S, k}\left[Q_{S, k}^{(1)} Q_{S, k}^{(2)}\right], \\
{\left[\begin{array}{c}
H_{R, k}^{(1)} \\
H_{R, k}^{(2)}
\end{array}\right] } & =\left[\begin{array}{l}
Q_{R, k}^{(1)} \\
Q_{R, k}^{(2)}
\end{array}\right] \Lambda_{R, k} V_{R, k}, \\
\Lambda_{t, k} & =\operatorname{diag}\left\{\sqrt{h_{t, k, n}}\right\}_{n=1}^{N}, t \in\{S, R\},
\end{aligned}
$$

where $V_{S, k}$ and $V_{R, k}$ are unitary matrices of dimension $N \times N ; h_{S, k, n}$ and $h_{R, k, n}$ are the eigenvalues of $H_{S, k}^{(1)}\left(H_{S, k}^{(1)}\right)^{H}+H_{S, k}^{(2)}\left(H_{S, k}^{(2)}\right)^{H}$ and $\left(H_{R, k}^{(1)}\right)^{H} H_{R, k}^{(1)}+$ $\left(H_{R, k}^{(2)}\right)^{H} H_{R, k}^{(2)}$, respectively. Matrices $Q_{S, k} \triangleq\left[Q_{S, k}^{(1)} Q_{S, k}^{(2)}\right]$ and $Q_{R, k} \triangleq\left[\begin{array}{l}Q_{R, k}^{(1)} \\ Q_{R, k}^{(2)}\end{array}\right]$ have orthonormal rows and orthonormal columns, respectively:

$$
\begin{aligned}
& Q_{S, k}^{(1)}\left(Q_{S, k}^{(1)}\right)^{H}+Q_{S, k}^{(2)}\left(Q_{S, k}^{(2)}\right)^{H}=I_{N}, \\
& \left(Q_{R, k}^{(1)}\right)^{H} Q_{R, k}^{(1)}+\left(Q_{R, k}^{(2)}\right)^{H} Q_{R, k}^{(2)}=I_{N} .
\end{aligned}
$$

As such, for

$$
\begin{aligned}
F_{k} & =V_{R, k}^{H} \boldsymbol{\Lambda}_{k} V_{S, k}^{H}, \quad \boldsymbol{\Lambda}_{k}=\operatorname{diag}\left\{\sqrt{\beta_{k, n}} \sqrt{\mathbf{p}_{R, k, n}}\right\}_{n=1}^{N} ; \\
\Psi_{k}^{(\ell)} & =\left(Q_{S, k}^{(\ell)}\right)^{-1} \boldsymbol{\Lambda}_{k}^{(\ell)}, \quad \boldsymbol{\Lambda}_{k}^{(\ell)}=\operatorname{diag}\left\{\sqrt{\mathbf{p}_{S, k, n}^{(\ell)}}\right\}_{n=1}^{N}, \ell \in\{1,2\}
\end{aligned}
$$

one can rewrite (2) as:

$$
\begin{aligned}
\tilde{y}_{D, k}^{(\ell)}= & \underbrace{Q_{R, k}^{(\ell)} \Lambda_{R, k} \boldsymbol{\Lambda}_{k} \Lambda_{S, k} \boldsymbol{\Lambda}_{k}^{(3-\ell)} s_{k}^{(3-\ell)}}_{\text {desired signal }}+\underbrace{Q_{R, k}^{(\ell)} \Lambda_{R, k} \boldsymbol{\Lambda}_{k} \Lambda_{S, k} \boldsymbol{\Lambda}_{k}^{(\ell)} s_{k}^{(\ell)}}_{\text {back-propagating self-interference }} \\
& +\underbrace{Q_{R, k}^{(\ell)} \Lambda_{R, k} \boldsymbol{\Lambda}_{k} V_{S, k}^{H} e_{\iota l, k}+e_{\mathrm{L}, k}^{(\ell)}}_{\text {FD loop interferences }}+\underbrace{Q_{R, k}^{(\ell)} \Lambda_{R, k} \boldsymbol{\Lambda}_{k} V_{S, k}^{H} \tilde{w}_{R, k}+\tilde{w}_{D, k}^{(\ell)},}_{\text {amplified noise }}
\end{aligned}
$$


or equivalently,

$$
\begin{aligned}
y_{D, k}^{(\ell)}= & \underbrace{\Lambda_{R, k} \boldsymbol{\Lambda}_{k} \Lambda_{S, k} \boldsymbol{\Lambda}_{k}^{(3-\ell)} s_{k}^{(3-\ell)}}_{\text {desired signal }}+\underbrace{\Lambda_{R, k} \boldsymbol{\Lambda}_{k} \Lambda_{S, k} \boldsymbol{\Lambda}_{k}^{(\ell)} s_{k}^{(\ell)}}_{\text {back-propagating self-interference }} \\
& +\underbrace{\Lambda_{R, k} \boldsymbol{\Lambda}_{k} t_{\mathrm{Ll}, k}+t_{\mathrm{Ll}, k}^{(\ell)}}_{\text {FD loop interferences }}+\underbrace{\Lambda_{R, k} \boldsymbol{\Lambda}_{k} w_{R, k}}_{\text {amplified noise }}+w_{D, k}^{(\ell)},
\end{aligned}
$$

where

$$
\begin{aligned}
& y_{D, k}^{(\ell)}=\left(Q_{R, k}^{(\ell)}\right)^{-1} \tilde{y}_{D, k}^{(\ell)}, \quad t_{\mathrm{L}, k}=V_{S, k}^{H} e_{L \mathrm{~L}, k}, \quad t_{\mathrm{Ll}, k}^{(\ell)}=\left(Q_{R, k}^{(\ell)}\right)^{-1} e_{\mathrm{Ll}, k}^{(\ell)}, \\
& w_{R, k}=V_{S, k}^{H} \tilde{w}_{R, k}, \quad w_{D, k}^{(\ell)}=\left(Q_{R, k}^{(\ell)}\right)^{-1} \tilde{w}_{D, k}^{(\ell)}, \ell \in\{1,2\} .
\end{aligned}
$$

The noises $w_{R, k}$ and $w_{D, k}^{(\ell)}$ are still zero-mean Gaussian with covariances ${ }^{1}$

$$
\begin{aligned}
& \mathcal{R}_{R, k}=V_{S, k}^{H} \mathcal{R}_{R} V_{S, k} \quad \text { and } \\
& \mathcal{R}_{D, k}^{(\ell)}=\left(Q_{R, k}^{(\ell)}\right)^{-1} \mathcal{R}_{D}^{(\ell)}\left(Q_{R, k}^{(\ell)}\right)^{-H}, \ell \in\{1,2\}
\end{aligned}
$$

respectively. Denoting $q_{k, n}^{(\ell)}$ as the diagonal entries of $\left(Q_{S, k}^{(\ell)}\right)^{-H}\left(Q_{S, k}^{(\ell)}\right)^{-1}$, the transmit power from user $\ell \in$ $\{1,2\}$ over subcarrier $k$ is then $\sum_{n=1}^{N} q_{k, n}^{(\ell)} \mathbf{p}_{S, k, n}^{(\ell)}$.

With (7), we have shown that the two-way relay MIMO channel in each OFDM subcarrier can be diagonalized into $N$ parallel channels, where the substraction of the known signals can also be performed on each spatial channel in each OFDM subcarrier. In (5), $\mathbf{p}_{R, k, n}$ and $\mathbf{p}_{S, k, n}^{(\ell)}$ are respectively the equivalent transmit power of the relay to the users and of the user $\ell \in\{1,2\}$ to the relay on spatial channel $n$ in subcarrier $k$. Note that the relaying power in $F_{k}$ is not amplified. In regard to the self-loop interferences in (8), it follows from $[5,26]$ that practically $\mathcal{E}\left\{\left\|t_{\mathrm{LI}, k}\right\|^{2}\right\} \leq h_{\mathrm{LI}} \sum_{n=1}^{N} \mathbf{p}_{R, k, n}$ and $\mathcal{E}\left\{\left\|t_{\mathrm{LI}, k}^{(\ell)}\right\|^{2}\right\} \leq$ $h_{\mathrm{LI}}^{(\ell)} \sum_{n=1}^{N} \mathbf{p}_{S, k, n}^{(\ell)}$ for some instantaneous residual self-loop interference powers $h_{\mathrm{LI}}$ and $h_{\mathrm{LI}}^{(\ell)}$. To have mathematically tractable formulations for power allocation, we make the following simplified assumption [17]:

$$
\begin{aligned}
& \mathcal{E}\left\{\left|t_{\mathrm{LI}, k}(n)\right|^{2}\right\} \leq h_{\mathrm{LI}, k, n} \mathbf{p}_{R, k, n} ; \\
& \mathcal{E}\left\{\left|t_{\mathrm{Ll}, k}^{(\ell)}(n)\right|^{2}\right\} \leq h_{\mathrm{LI}, k, n}^{S} \mathbf{p}_{S, k, n}^{(\ell)}, \ell \in\{1,2\}
\end{aligned}
$$

where $h_{\mathrm{LI}, k, n}$ and $h_{\mathrm{LI}, k, n}^{S}$ represent the instantaneous residual self-loop interference powers of spatial channel $n$ on subcarrier $k$ at the relay and at the users, respectively.
Therefore, the amplify gain $\beta_{k, n}$ in (5) to guarantee the transmit power $\mathbf{p}_{R, k, n}$ of each channel $n$ on subcarrier $k$ is

$$
\begin{aligned}
\beta_{k, n} & =1 / \sqrt{h_{S, k, n}\left(\mathbf{p}_{S, k, n}^{(1)}+\mathbf{p}_{S, k, n}^{(2)}\right)+h_{L l, k, n} \mathbf{p}_{R, k, n}+\mathcal{R}_{R, k}(n, n)} \\
& =\sqrt{\frac{1 / \mathcal{R}_{R, k}(n, n)\left(\gamma_{L \iota, k, n} \mathbf{p}_{R, k, n}+1\right)}{\bar{h}_{S, k, n} \gamma_{k, n}\left(\mathbf{p}_{S, k, n}^{(1)}+\mathbf{p}_{S, k, n}^{(2)}, \mathbf{p}_{R, k, n}\right)+1}},
\end{aligned}
$$

where we define $\bar{h}_{S, k, n} \triangleq h_{S, k, n} / \mathcal{R}_{R, k}(n, n), \quad \ell \in\{1,2\}$; $\gamma_{\mathrm{LI}, k, n} \triangleq h_{\mathrm{LI}, k, n} / \mathcal{R}_{R, k}(n, n)$ and

$$
\gamma_{k, n}\left(\mathbf{p}_{S, k, n}^{(1)}+\mathbf{p}_{S, k, n}^{(2)}, \mathbf{p}_{R, k, n}\right) \triangleq \frac{\mathbf{p}_{S, k, n}^{(1)}+\mathbf{p}_{S, k, n}^{(1)}}{\gamma_{\mathrm{Ll}, k, n} \mathbf{p}_{R, k, n}+1}, \ell \in\{1,2\} .
$$

After subtracting the back-propagating signal in (7), the instantaneous SNR at user $\ell$ over spatial channel $n$ of subcarrier $k$ is

$$
\begin{aligned}
& \operatorname{SNR}_{k, n}^{(\ell)} \\
& =\frac{\bar{h}_{S, k, n} \gamma_{k, n}\left(\mathbf{p}_{S, k, n}^{(3-\ell)}, \mathbf{p}_{R, k, n}\right) \bar{h}_{R, k, n}^{(\ell)} \xi_{k, n}^{(\ell)}\left(\mathbf{p}_{R, k, n}, \mathbf{p}_{S, k, n}^{(\ell)}\right)}{1+\bar{h}_{S, k, n} \gamma_{k, n}\left(\mathbf{p}_{S, k, n}^{(1)}+\mathbf{p}_{S, k, n}^{(2)}, \mathbf{p}_{R, k, n}\right)+\bar{h}_{R, k, n}^{(\ell)} \xi_{k, n}^{(\ell)}\left(\mathbf{p}_{R, k, n}, \mathbf{p}_{S, k, n}^{(\ell)}\right)}
\end{aligned}
$$

where we define $\gamma_{k, n}\left(\mathbf{p}_{S, k, n}^{(3-\ell)}, \mathbf{p}_{R, k, n}\right) \triangleq \mathbf{p}_{S, k, n}^{(3-\ell)} /$ $\left(\gamma_{\mathrm{LI}, k, n} \mathbf{p}_{R, k, n}+1\right), \quad \bar{h}_{R, k, n}^{(\ell)} \triangleq h_{R, k, n} / \mathcal{R}_{D, k}^{(\ell)}(n, n), \quad$ and $\xi_{k, n}^{(\ell)}\left(\mathbf{p}_{R, k, n}, \mathbf{p}_{S, k, n}^{(\ell)}\right) \triangleq \mathbf{p}_{R, k, n} /\left(\gamma_{\mathrm{Ll}, k, n}^{(\ell)} \mathbf{p}_{S, k, n}^{(\ell)}+1\right)$ with $\gamma_{\mathrm{L}, k, n}^{(\ell)} \triangleq h_{\mathrm{LL}, k, n}^{S} / \mathcal{R}_{D, k}^{(\ell)}(n, n)$.

Upon defining

$a_{(k-1) N+n} \triangleq \bar{h}_{S, k, n} ; \quad b_{(k-1) N+n}^{(\ell)} \triangleq \bar{h}_{R, k, n}^{(\ell)}, \quad \ell \in\{1,2\} ;$

$\gamma_{\mathrm{LI},(k-1) N+n} \triangleq \gamma_{\mathrm{LI}, k, n} ; \quad \gamma_{\mathrm{Ll},(k-1) N+n}^{(\ell)} \triangleq \gamma_{\mathrm{Ll}, k, n^{\prime}}^{(\ell)}$

$q_{(k-1) N+n}^{(\ell)} \triangleq q_{k, n}^{(\ell)}, \quad \mathbf{x}_{(k-1) N+n}^{(\ell)} \triangleq \mathbf{p}_{S, k, n}^{(\ell)}, \quad \ell \in\{1,2\} ;$

$\mathbf{y}_{(k-1) N+n} \triangleq \mathbf{p}_{R, k, n} ; \quad \gamma_{(k-1) N+n}\left(\mathbf{x}_{(k-1) N+n}^{(\ell)}, \mathbf{y}_{(k-1) N+n}\right)$

$$
\triangleq \gamma_{k, n}\left(\mathbf{p}_{S, k, n}^{(\ell)}, \mathbf{p}_{R, k, n}\right) \text {, }
$$

$\xi_{(k-1) N+n}^{(\ell)}\left(\mathbf{y}_{(k-1) N+n}, \mathbf{x}_{(k-1) N+n}^{(\ell)}\right) \triangleq \xi_{k, n}^{(\ell)}\left(\mathbf{p}_{R, k, n}, \mathbf{p}_{S, k, n}^{(\ell)}\right)$,

$\mathbf{x}^{(\ell)} \triangleq\left(\mathbf{x}_{1}^{(\ell)}, \ldots, \mathbf{x}_{K N}^{(\ell)}\right)^{T}, \ell \in\{1,2\} ; \quad \mathbf{y} \triangleq\left(\mathbf{y}_{1}, \ldots, \mathbf{y}_{K N}\right)^{T} ;$

$\mathbf{x} \triangleq\left(\mathbf{x}^{(1)}, \mathbf{x}^{(2)}\right) ;$

and $M \triangleq K N$, we are concerned with the problem of maximizing the network instantaneous throughput under transmit power constraints. The optimization problem is formulated as ${ }^{2}$ :

$\max _{(\mathbf{x}, \mathbf{y})} \sum_{i=1}^{M} \sum_{\ell=1}^{2} \ln \left(1+\frac{a_{i} \gamma_{i}\left(\mathbf{x}_{i}^{(3-\ell)}, \mathbf{y}_{i}\right) b_{i}^{(\ell)} \xi_{i}^{(\ell)}\left(\mathbf{y}_{i}, \mathbf{x}_{i}^{(\ell)}\right)}{1+a_{i} \gamma_{i}\left(\mathbf{x}_{i}^{(1)}+\mathbf{x}_{i}^{(2)}, \mathbf{y}_{i}\right)+b_{i}^{(\ell)} \xi_{i}^{(\ell)}\left(\mathbf{y}_{i}, \mathbf{x}_{i}^{(\ell)}\right)}\right)$ 
subject to the joint sum-power constraint

$$
\sum_{i=1}^{M}\left(q_{i}^{(1)} \mathbf{x}_{i}^{(1)}+q_{i}^{(2)} \mathbf{x}_{i}^{(2)}+\mathbf{y}_{i}\right) \leq P
$$

or to the separate sum-power constraints at each node

$$
\sum_{i=1}^{M} q_{i}^{(\ell)} \mathbf{x}_{i}^{(\ell)} \leq P_{1}^{(\ell)}, \ell \in\{1,2\} ; \quad \sum_{i=1}^{M} \mathbf{y}_{i} \leq P_{2} .
$$

Here, $P \geq 0, P_{1}^{(\ell)} \geq 0, P_{2} \geq 0$ are the predefined power budgets. In practice, the users and the relay have separate power supplies constrained by (16) and the power allocation is performed at individual nodes. However, it is also important to consider the joint power allocation with the total power constraint (15) to gain meaningful insights into the power utilization of the whole system, and thereby realizing its full capacity.

In the HD two-way relaying strategy, the MA and $\mathrm{BC}$ phases are consecutively processed. There is no selfloop interference at the relay and users in this case (i.e., $\left.\gamma_{\mathrm{LL}, k, n}=\gamma_{\mathrm{L}, k, n}^{S} \equiv 0\right)$; and thus, $\gamma_{i}\left(\mathbf{x}_{i}^{(3-\ell)}, \mathbf{y}_{i}\right)=\mathbf{x}_{i}^{(3-\ell)}$ and $\xi_{i}^{(\ell)}\left(\mathbf{y}_{i}, \mathbf{x}_{i}^{(\ell)}\right)=\mathbf{y}_{i}$. The instantaneous total throughput maximization in (14) reduces to:

$$
\max _{(\mathbf{x}, \mathbf{y})} \frac{1}{2} \sum_{i=1}^{M} \sum_{\ell=1}^{2} \ln \left(1+\frac{a_{i} \mathbf{x}_{i}^{(3-\ell)} b_{i}^{(\ell)} \mathbf{y}_{i}}{1+a_{i}\left(\mathbf{x}_{i}^{(1)}+\mathbf{x}_{i}^{(2)}\right)+b_{i}^{(\ell)} \mathbf{y}_{i}}\right)
$$

subject to either (15) or (16). In (17), the pre-log factor of $1 / 2$ accounts for the two time slots needed to transmit one data packet. Note that joint power allocation in nonregenerative MIMO two-way relaying has previously been considered, e.g., in [27], under the reciprocity assumption $H_{R, k}^{(\ell)}=\left(H_{S, k}^{(\ell)}\right)^{H}, \ell \in\{1,2\}$, in which the corresponding channel (2) (with no amplified self-loop interference) is parallelized by precoding the data symbol $s_{k}^{(\ell)}$ for subchannel alignment.

\subsection{MIMO-OFDM FD One-Way Relaying}

The above two-way relaying communications allow two data packets to be transmitted in opposite directions between user $\ell \in\{1,2\}$ and user $3-\ell$ in either one time slot (i.e., using FD mode) or two time slots (i.e., using HD mode). In one-way relaying communications, there are no $\mathrm{MA}$ and $\mathrm{BC}$ phases. In the $\mathrm{FD}$ one-way relaying strategy, user $\ell \in\{1,2\}$ sends one data packet to user $3-\ell$ through the relay in one time slot. There is no self-loop interference at user $3-\ell$, i.e., $\gamma_{\mathrm{Ll}, k, n}^{(3-\ell)} \equiv 0$. Considering user $\ell$, instead of SVD (3), one uses the SVDs $H_{S, k}^{(\ell)}=V_{S, k}^{(\ell)} \Lambda_{S, k}^{(\ell)} U_{S, k}^{(\ell)}$ and $H_{R, k}^{(3-\ell)}=U_{R, k}^{(\ell)} \Lambda_{R, k}^{(\ell)} V_{R, k}^{(\ell)}$ with unitary matrices $U_{t, k}^{(\ell)}$ and $V_{t, k}^{(\ell)}, t \in\{S, R\}$ and diagonal matrices $\Lambda_{t, k}^{(\ell)}=\operatorname{diag}\left\{\sqrt{h_{t, k, n}^{(\ell)}}\right\}_{n=1}^{N}, t \in\{S, R\}$. Accordingly, $F_{k}^{(\ell)}=\left(V_{R, k}^{(\ell)}\right)^{H} \Lambda^{(\ell)}\left(V_{S, k}^{(\ell)}\right)^{H}$ while the covariance $\mathcal{R}_{R, k}$ are the same as that in (9) and $Q_{R, k}^{(3-\ell)}=U_{R, k}^{(\ell)}$ for defining the covariance $\mathcal{R}_{D, k}^{(3-\ell)}$ in (9). Now, let us set

$$
\begin{aligned}
a_{(k-1) N+n}^{(\ell)} & \triangleq h_{S, k, n}^{(\ell)}, \quad b_{(k-1) N+n}^{(\ell)} \triangleq h_{R, k, n}^{(\ell)}, \\
\mathbf{x}^{(\ell)} & \triangleq\left(\mathbf{x}_{1}^{(\ell)}, \ldots, \mathbf{x}_{K N}^{(\ell)}\right)^{T}, \\
\mathbf{y}^{(\ell)} & \triangleq\left(\mathbf{y}_{1}^{(\ell)}, \ldots, \mathbf{y}_{K N}^{(\ell)}\right)^{T}, \ell \in\{1,2\} .
\end{aligned}
$$

Then instead of (14), the one-way instantaneous sum throughput maximization is

$\max _{\left(\mathbf{x}^{(\ell)}, \mathbf{y}^{(\ell)}\right), \ell \in\{1,2\}} \frac{1}{2} \sum_{i=1}^{M} \sum_{\ell=1}^{2} \ln \left(1+\frac{a_{i}^{(\ell)} \gamma_{i}\left(\mathbf{x}_{i}^{(\ell)}, \mathbf{y}_{i}^{(\ell)}\right) b_{i}^{(\ell)} \mathbf{y}_{i}^{(\ell)}}{1+a_{i}^{(\ell)} \gamma_{i}\left(\mathbf{x}_{i}^{(\ell)}, \mathbf{y}_{i}^{(\ell)}\right)+b_{i}^{(\ell)} \mathbf{y}_{i}^{(\ell)}}\right)$

subject to the joint sum-power constraint

$$
\sum_{i=1}^{M}\left(\mathbf{x}_{i}^{(\ell)}+\mathbf{y}_{i}^{(\ell)}\right) \leq P / 2, \ell \in\{1,2\}
$$

or to the separate sum-power constraints

$$
\sum_{i=1}^{M} \mathbf{x}_{i}^{(\ell)} \leq P_{1}^{(\ell)}, \quad \sum_{i=1}^{M} \mathbf{y}_{i}^{(\ell)} \leq P_{2} / 2, \ell \in\{1,2\} .
$$

In (18), the pre-log factor of $1 / 2$ accounts for the two time slots needed to transmit one data packet from user $\ell$ to user $3-\ell$ and then another data packet from user $3-\ell$ to user $\ell$. In (19) and (20), the power budgets $P / 2$ and $P_{2} / 2$ are used to facilitate a fair performance comparison between one-way relaying and two-way relaying. Note that the problem (18) s.t. (19) was previously considered in [17] under the additional assumption that both the noises $\tilde{w}_{R, k}$ and $\tilde{w}_{D}^{(\ell)}$ are white. Note also that (18) can be decomposed into two independent sum throughput maximization problems as:

$$
\max _{\left(\mathbf{x}^{(\ell)}, \mathbf{y}^{(\ell)}\right)} \frac{1}{2} \sum_{i=1}^{M} \ln \left(1+\frac{a_{i}^{(\ell)} \gamma_{i}\left(\mathbf{x}_{i}^{(\ell)}, \mathbf{y}_{i}^{(\ell)}\right) b_{i}^{(\ell)} \mathbf{y}_{i}^{(\ell)}}{1+a_{i}^{(\ell)} \gamma_{i}\left(\mathbf{x}_{i}^{(\ell)}, \mathbf{y}_{i}^{(\ell)}\right)+b_{i}^{(\ell)} \mathbf{y}_{i}^{(\ell)}}\right)
$$$$
\text { s.t. }(19) /(20) \text {, }
$$

for $\ell \in\{1,2\}$.

With the HD one-way relaying strategy, two time slots are used to send one data packet from one user to another user via the relay. Since $\gamma\left(\mathbf{x}_{i}^{(\ell)}, \mathbf{y}_{i}\right)=\mathbf{x}_{i}^{(\ell)}$, we have the 
following one-way instantaneous sum-rate maximization per time slot [16]:

$$
\max _{\left(\mathbf{x}^{(\ell)}, \mathbf{y}^{(\ell)}\right), \ell \in\{1,2\}} \frac{1}{4} \sum_{i=1}^{M} \sum_{\ell=1}^{2} \ln \left(1+\frac{a_{i}^{(\ell)} \mathbf{x}_{i}^{(\ell)} b_{i}^{(\ell)} \mathbf{y}_{i}^{(\ell)}}{1+a_{i}^{(\ell)} \mathbf{x}_{i}^{(\ell)}+b_{i}^{(\ell)} \mathbf{y}_{i}^{(\ell)}}\right)
$$

subject to (19) or (20), which can be decomposed into two independent sum throughput maximization problems as:

$$
\begin{aligned}
\max _{\left(\mathbf{x}^{(\ell)}, \mathbf{y}^{(\ell)}\right)} & \frac{1}{4} \sum_{i=1}^{M} \ln \left(1+\frac{a_{i}^{(\ell)} \mathbf{x}_{i}^{(\ell)} b_{i}^{(\ell)} \mathbf{y}_{i}^{(\ell)}}{1+a_{i}^{(\ell)} \mathbf{x}_{i}^{(\ell)}+b_{i}^{(\ell)} \mathbf{y}_{i}^{(\ell)}}\right) \\
\text { s.t. } & (19) /(20),
\end{aligned}
$$

for $\ell \in\{1,2\}$.

This paper focuses on solving the general FD twoway/one-way relaying problems (14) s.t. (15)/(16) and (18) s.t. $(19) /(20)$. The solutions to the HD two-way/one-way relaying problems (17) s.t. (15)/(16) and (22) s.t. (19)/(20) follow directly by replacing $\gamma \mathrm{LI}, i=0$, and will be used as benchmarks for performance comparison between the FD and $\mathrm{HD}$ relaying strategies.

\section{Proposed Solutions for Two-Way Relaying}

To the best of our knowledge, the FD two-way relaying problems (14) s.t. (15)/(16) have never been considered before. From the definition of the fractional function $\gamma_{i}\left(\mathbf{x}_{i}^{(\ell)}, \mathbf{y}_{i}\right)$ in (12), it is clear that the objective in (14) is a very complex nonconcave function with many fractional and cross terms in the source power variables $\mathbf{x} \triangleq$ $\left(\mathbf{x}^{(1)}, \mathbf{x}^{(2)}\right)$ and the relay variable $\mathbf{y}$. Moreover, this objective function is not individually concave in either $\mathbf{x}$ or $\mathbf{y}$. Even performing the power allocation either at source node only or at the relay node only is already difficult. Although the objective function in (17) is simpler than (14), the same challenge remains in the HD two-way relaying problems (17) s.t. (15)/(16).

For ease of reference, we present below the properties that will be frequently used in our solution development.

$$
\begin{array}{ll}
- & (\mathbf{P} 1): \ln \left(x_{1}+x_{2}\right) \leq \ln \left(x_{1}^{(0)}+x_{2}^{(0)}\right)+ \\
& \frac{1}{x_{1}^{(0)}+x_{2}^{(0)}}\left[\left(x_{1}-x_{1}^{(0)}\right)+\left(x_{2}-x_{2}^{(0)}\right)\right] \text { for all } \\
& x_{1}>0, x_{2} \geq 0, x_{1}^{(0)}>0, x_{2}^{(0)} \geq 0 . \\
- & (\mathbf{P} 2): \ln \left(x_{1}+x_{2}\right) \geq \ln \left(x_{1}^{(0)}+x_{2}^{(0)}\right)+ \\
& \frac{1}{x_{1}^{(0)}+x_{2}^{(0)}}\left[x_{1}^{(0)}\left(\ln x_{1}-\ln x_{1}^{(0)}\right)+\right. \\
& \left.x_{2}^{(0)}\left(\ln x_{2}-\ln x_{2}^{(0)}\right)\right] \text { for all } \\
& x_{1}>0, x_{2}>0, x_{1}^{(0)}>0, x_{2}^{(0)}>0 .
\end{array}
$$

Property (P1) is the following well-known property of the concave function $h\left(x_{1}, x_{2}\right) \triangleq \ln \left(x_{1}+x_{2}\right)$ [28]:

$\left.h\left(x_{1}, x_{2}\right) \leq h\left(x_{1}^{(0)}+x_{2}^{(0)}\right)+\left\langle\nabla h\left(x_{1}^{(0)}, x_{2}^{(0)}\right), x_{1}, x_{2}\right)-\left(x_{1}^{(0)}, x_{2}^{(0)}\right)\right\rangle$, $\forall x_{1}>0, x_{2} \geq 0, x_{1}^{(0)}>0, x_{2}^{(0)} \geq 0$. On the other hand, the function $\tilde{h}\left(\tilde{x}_{1}, \tilde{x}_{2}\right) \triangleq \ln \left(e^{\tilde{x}_{1}}+e^{\tilde{x}_{2}}\right)$ is convex in $\left(\tilde{x}_{1}, \tilde{x}_{2}\right)$ and so $[28]$

$$
\begin{gathered}
\tilde{h}\left(\tilde{x}_{1}, \tilde{x}_{2}\right) \geq \tilde{h}\left(\tilde{x}_{1}^{(0)}, \tilde{x}_{2}^{(0)}\right)+\left\langle\nabla \tilde{h}\left(\tilde{x}_{1}^{(0)}, \tilde{x}_{2}^{(0)}\right),\right. \\
\left.\left(\tilde{x}_{1}, \tilde{x}_{2}\right)-\left(\tilde{x}_{1}^{(0)}, \tilde{x}_{2}^{(0)}\right)\right\rangle
\end{gathered}
$$

$\forall \tilde{x}_{1}, \tilde{x}_{2}, \tilde{x}_{1}^{(0)}, \tilde{x}_{2}^{(0)}$. Upon noting that

$$
\nabla \tilde{h}\left(\tilde{x}_{1}^{(0)}, \tilde{x}_{2}^{(0)}\right)=\frac{1}{e^{\tilde{x}_{1}^{(0)}}+e^{\tilde{x}_{2}^{(0)}}}\left(e^{\tilde{x}_{1}^{(0)}}, e^{\tilde{x}_{2}^{(0)}}\right)^{T},
$$

property (P2) then follows after replacing $x_{i}=e^{\tilde{x}_{i}}, x_{i}^{(0)}=$ $e^{\tilde{x}_{i}^{(0)}}, i \in\{1,2\}$ in (24) and (25). This property is the key for the success of the SCALE algorithm in the multiuser OFDM spectrum balancing problem [29].

All problems considered in this paper can be expressed in the following form:

$$
\max _{(\mathbf{x}, \mathbf{y})} F(\mathbf{x}, \mathbf{y}) \quad \text { s.t. } \quad(\mathbf{x}, \mathbf{y}) \in \mathcal{S},
$$

where $F(\mathbf{x}, \mathbf{y})$ is not a concave function and $\mathcal{S}$ is a convex set defined by either $(15) /(16)$ or $(19) /(20)$. By adopting the inner approximation method (see, e.g., [30]), our strategy is to iteratively approximate function $F(\cdot)$ by a concave function $F^{(\kappa)}(\cdot)$ that possesses the following two crucial properties:

- It matches the nonconcave objective function $F(\cdot)$ at $\left(x^{(\kappa)}, y^{(\kappa)}\right)$, i.e.,

$$
F^{(\kappa)}\left(x^{(\kappa)}, y^{(\kappa)}\right)=F\left(x^{(\kappa)}, y^{(\kappa)}\right) .
$$

- It is a global lower bound of the nonconcave objective function $F(\cdot)$, i.e.,

$$
F^{(\kappa)}(\mathbf{x}, \mathbf{y}) \leq F(\mathbf{x}, \mathbf{y}), \forall(\mathbf{x}, \mathbf{y}) .
$$

These properties guarantee that $F^{(\kappa)}(\cdot)$ is both a local and a global concave approximation of $F(\cdot)$ at $\left(x^{(\kappa)}, y^{(\kappa)}\right)$. A proximity control is therefore not necessary.

GENERIC ALGORITHM 1. Initialized by a feasible solution $\left(x^{(0)}, y^{(0)}\right)$ to problem (26), generate a feasible solution $\left(x^{(\kappa+1)}, y^{(\kappa+1)}\right)$ at $\kappa$-iteration for $\kappa=0,1, \ldots$, as the optimal solution of the following convex program:

$$
\max _{(\mathbf{x}, \mathbf{y})} F^{(\kappa)}(\mathbf{x}, \mathbf{y}) \quad \text { s.t. } \quad(\mathbf{x}, \mathbf{y}) \in \mathcal{S} .
$$

The convex program (29) provides an iterative minorant maximization for nonconvex program (26). Since $\left(x^{(\kappa)}, y^{(\kappa)}\right)$ is feasible to problem (29) itself, it follows that

$$
\begin{aligned}
F\left(x^{(\kappa)}, y^{(\kappa)}\right) & =F^{(\kappa)}\left(x^{(\kappa)}, y^{(\kappa)}\right)<F^{(\kappa)}\left(x^{(\kappa+1)}, y^{(\kappa+1)}\right) \\
& \leq F\left(x^{(\kappa+1)}, y^{(\kappa+1)}\right)
\end{aligned}
$$


as long as $\left(x^{(\kappa+1)}, y^{(\kappa+1)}\right) \neq\left(x^{(\kappa)}, y^{(\kappa)}\right)$. In other words, $\left(x^{(\kappa+1)}, y^{(\kappa+1)}\right)$ is a better solution of the nonconvex program (26) than $\left(x^{(\kappa)}, y^{(\kappa)}\right)$. Moreover, the necessary optimality condition for $\left(x^{(\kappa)}, y^{(\kappa)}\right)$ is $\left(x^{(\kappa+1)}, y^{(\kappa+1)}\right)=$ $\left(x^{(\kappa)}, y^{(\kappa)}\right)$. That is, for $\left(x^{(\kappa)}, y^{(\kappa)}\right)$ to be an optimal solution of the nonconvex program (26), it is necessary that $\left(x^{(\kappa)}, y^{(\kappa)}\right)$ is a globally optimal solution of the convex program (29). The efficiency is Algorithm 1 therefore hinges upon the computational tractability of (29).

Proposition 1 ([30]) For any function $F^{(\kappa)}(\cdot)$ satisfying the matching condition (27) and the lower bounding condition (28), the generic Algorithm 1 generates a sequence $\left\{\left(x^{(\kappa)}, y^{(\kappa)}\right)\right\}$ of improved solutions, which converges to at least a locally optimal solution of problem (26). Given a tolerance $\epsilon>0$, Algorithm 1 therefore terminates after a finite number of iterations under the stopping criterion

$$
F\left(x^{(\kappa+1)}, y^{(\kappa+1)}\right)-F\left(x^{(\kappa)}, y^{(\kappa)}\right)<\epsilon .
$$

In the two-way relaying problems (14) s.t. (15)/(16) and (17) s.t. (15)/(16), the throughput expression is complicated which in the end may only bring marginally higher performance than that by a tractable approximation. As such, we propose replacing the term $b_{i}^{(\ell)} \xi_{i}^{(\ell)}\left(\mathbf{y}_{i}, \mathbf{x}_{i}^{(\ell)}\right)$ (which is smaller than $b_{i}^{(\ell)} \mathbf{y}_{i}$ ) in the denominator of (14) by $b_{i}^{(\ell)} \mathbf{y}_{i}$ to obtain a tight lower bound for throughput. Then, we apply property (P2) to get the following lower bound at $\left(x^{(1, \kappa)}, x^{(1, \kappa)}, y^{(\kappa)}\right)$ for the objective in $(14)^{3}$ :

$$
\begin{aligned}
& \ln \left(1+\frac{a_{i} \gamma_{i}\left(\mathbf{x}_{i}^{(3-\ell)}, \mathbf{y}_{i}\right) b_{i}^{(\ell)} \xi_{i}^{(\ell)}\left(\mathbf{y}_{i}, \mathbf{x}_{i}^{(\ell)}\right)}{1+a_{i} \gamma\left(\mathbf{x}_{i}^{(1)}+\mathbf{x}_{i}^{(2)}, \mathbf{y}_{i}\right)+b_{i}^{(\ell)} \mathbf{y}_{i}}\right) \\
& \geq \alpha_{i}^{(\ell)}(\kappa) \ln \left(\frac{a_{i} \gamma_{i}\left(\mathbf{x}_{i}^{(3-\ell)}, \mathbf{y}_{i}\right) b_{i}^{(\ell)} \xi_{i}^{(\ell)}\left(\mathbf{y}_{i}, \mathbf{x}_{i}^{(\ell)}\right)}{1+a_{i} \gamma_{i}\left(\mathbf{x}_{i}^{(1)}+\mathbf{x}_{i}^{(2)}, \mathbf{y}_{i}\right)+b_{i}^{(\ell)} \mathbf{y}_{i}}\right)+\beta_{i}^{(\ell)}(\kappa),
\end{aligned}
$$

where

$$
\begin{aligned}
& \alpha_{i}^{(\ell)}(\kappa) \triangleq 1-\frac{1}{1+f_{i}^{(\ell)}(\kappa)}, \\
& \beta_{i}^{(\ell)}(\kappa) \triangleq \ln \left(1+f_{i}^{(\ell)}(\kappa)\right)-\left(1-\frac{1}{1+f_{i}^{(\ell)}(\kappa)}\right) \ln \left(f_{i}^{(\ell)}(\kappa)\right),
\end{aligned}
$$

$$
f_{i}^{(\ell)}(\kappa) \triangleq \frac{a_{i} \gamma_{i}\left(x_{i}^{(3-\ell, \kappa)}, y_{i}^{(\kappa)}\right) b_{i}^{(\ell)} \xi_{i}^{(\ell)}\left(y_{i}^{(\kappa)}, x_{i}^{(\ell, \kappa)}\right)}{1+a_{i} \gamma\left(x_{i}^{(1, \kappa)}+x_{i}^{(2, \kappa)}, y_{i}^{(\kappa)}\right)+b_{i}^{(\ell)} y_{i}^{(\kappa)}} .
$$

The $\kappa$-iteration of the generic Algorithm 1 is thus to solve the following problem in $\mathbf{x} \triangleq\left(\mathbf{x}^{(1)}, \mathbf{x}^{(2)}\right)$ and $\mathbf{y}$ :

$$
\max _{(\mathbf{x}, \mathbf{y})} F^{(\kappa)}(\mathbf{x}, \mathbf{y}) \quad \text { s.t. } \quad(15) /(16)
$$

with

$$
\begin{gathered}
F(\mathbf{x}, \mathbf{y}) \geq \sum_{i=1}^{M} \sum_{\ell=1}^{2}\left[\alpha_{i}^{(\ell)}(\kappa) \ln \left(\frac{a_{i} \gamma_{i}\left(\mathbf{x}_{i}^{(3-\ell)}, \mathbf{y}_{i}\right) b_{i}^{(\ell)} \xi_{i}^{(\ell)}\left(\mathbf{y}_{i}, \mathbf{x}_{i}^{(\ell)}\right)}{1+a_{i} \gamma\left(\mathbf{x}_{i}^{(1)}+\mathbf{x}_{i}^{(2)}, \mathbf{y}_{i}\right)+b_{i}^{(\ell)} \mathbf{y}_{i}}\right)\right. \\
\left.+\beta_{i}^{(\ell)}(\kappa)\right] \\
=\sum_{i=1}^{M} \sum_{\ell=1}^{2}\left[\alpha _ { i } ^ { ( \ell ) } ( \kappa ) \left(\ln \mathbf{x}_{i}^{(3-\ell)}+\ln \mathbf{y}_{i}-\ln \left(\gamma_{\mathrm{L}, i}^{(\ell)} \mathbf{x}_{i}^{(\ell)}+1\right)\right.\right. \\
-\ln \left(1+a_{i}\left(\mathbf{x}_{i}^{(1)}+\mathbf{x}_{i}^{(2)}\right)+\left(b_{i}^{(\ell)}+\gamma \mathrm{Ll}, i\right) \mathbf{y}_{i}+\gamma \mathrm{Ll}, b_{i}^{(\ell)} \mathbf{y}_{i}^{2}\right) \\
\left.\left.\quad+\ln \left(a_{i} b_{i}^{(\ell)}\right)\right)+\beta_{i}^{(\ell)}(\kappa)\right] \\
\geq \sum_{i=1}^{M} \sum_{\ell=1}^{2}\left[\alpha _ { i } ^ { ( \ell ) } ( \kappa ) \left(\ln \mathbf{x}_{i}^{(3-\ell)}+\ln \mathbf{y}_{i}-d_{i}^{(\ell, \kappa)}\left(\mathbf{x}_{i}^{(\ell)}-x_{i}^{(\ell, \kappa)}\right)\right.\right. \\
-c_{i}^{(\ell, \kappa)}\left(a_{i}\left(\mathbf{x}_{i}^{(1)}-x_{i}^{(1, \kappa)}\right)+a_{i}\left(\mathbf{x}_{i}^{(2)}-x_{i}^{(2, \kappa)}\right)\right. \\
\left.+\left(b_{i}^{(\ell)}+\gamma \mathrm{Ll}, i\right)\left(\mathbf{y}_{i}-y_{i}^{(\kappa)}\right)+\gamma \mathrm{Ll}, b_{i}^{(\ell)}\left(y_{i}^{2}-\left(y_{i}^{(\kappa)}\right)^{2}\right)\right) \\
\left.\left.+\ln \left(a_{i} b_{i}^{(\ell)}\right)\right)-\ln \left(\gamma_{\mathrm{L}, i}^{(\ell)} x_{i}^{(\ell, \kappa)}+1\right)+\ln c_{i}^{(\ell, \kappa)}+\beta_{i}^{(\ell)}(\kappa)\right]
\end{gathered}
$$

and

$$
\begin{aligned}
& d_{i}^{(\ell, \kappa)} \triangleq \frac{\gamma_{\mathrm{L}, i}^{(\ell)}}{\gamma_{\mathrm{L}, i}^{(\ell)} x_{i}^{(\ell, \kappa)}+1}, \\
& c_{i}^{(\ell, \kappa)} \triangleq \frac{1}{1+a_{i}\left(x_{i}^{(1, \kappa)}+x_{i}^{(2, \kappa)}\right)+\left(b_{i}^{(\ell)}+\gamma_{\mathrm{L}, i, i}\right) y_{i}^{(\kappa)}+\gamma_{\mathrm{L}, i} b_{i}^{(\ell)}\left(y_{i}^{(\kappa)}\right)^{2}} .
\end{aligned}
$$

The inequality (35) follows from property (P2) whereas (36) from property (P1).

It is worth mentioning that the function $F^{(\kappa)}(\mathbf{x}, \mathbf{y})$ satisfies the two crucial properties (27) and (28) for an effective implementation of the generic Algorithm 1. In the case of constraint (15), the KKT conditions for necessary and sufficient optimality of the convex program (34) s.t. (15) are

$$
\begin{gathered}
\frac{\alpha_{i}^{(3-\ell)}(\kappa)}{\mathbf{x}_{i}^{(\ell)}}-\alpha_{i}^{(\ell)}(\kappa) d_{i}^{(\ell, \kappa)}-\sum_{t=1}^{2} \alpha_{i}^{(t)}(\kappa) a_{i} c_{i}^{(t, \kappa)} \\
-\lambda q_{i}^{(\ell)}=0, \ell \in\{1,2\}, \\
\frac{\sum_{t=1}^{2} \alpha_{i}^{(t)}(\kappa)}{\mathbf{y}_{i}}-\sum_{t=1}^{2} \alpha_{i}^{(t)}(\kappa) c_{i}^{(t, \kappa)}\left(b_{i}^{(t)}+\gamma \mathrm{LI}, i+2 \gamma \mathrm{Ll}, b_{i}^{(t)} \mathbf{y}_{i}\right) \\
-\lambda=0 .
\end{gathered}
$$

Therefore, the solution for problem (34) s.t. (15) is derived as:

$$
x_{i}^{(\ell, \kappa+1)}=\frac{\alpha_{i}^{(3-\ell)}(\kappa)}{\alpha_{i}^{(\ell)}(\kappa) d_{i}^{(\ell, \kappa)}+\sum_{t=1}^{2} \alpha_{i}^{(t)}(\kappa) a_{i} c_{i}^{(t, \kappa)}+\lambda q_{i}^{(\ell)}}, \ell \in\{1,2\},
$$




$$
y_{i}^{(\kappa+1)}=\frac{2 p_{i}(\kappa)}{r_{i}(\kappa)+\lambda+\sqrt{\left(r_{i}(\kappa)+\lambda\right)^{2}+4 p_{i}(\kappa) q_{i}(\kappa)}},
$$

where

$$
\begin{aligned}
& p_{i}(\kappa) \triangleq \sum_{t=1}^{2} \alpha_{i}^{(t)}(\kappa), \\
& q_{i}(\kappa) \triangleq 2 \gamma_{\mathrm{L}, i} \sum_{t=1}^{2} \alpha_{i}^{(t)}(\kappa) c_{i}^{(t, \kappa)} b_{i}^{(t)}, \\
& r_{i}(\kappa) \triangleq \sum_{t=1}^{2} \alpha_{i}^{(t)}(\kappa) c_{i}^{(t, \kappa)}\left(b_{i}^{(t)}+\gamma_{\mathrm{LL}, i}\right),
\end{aligned}
$$

and $\lambda>0$ is chosen such that $\left(x^{(1, \kappa+1)}, x^{(2, \kappa+1)}, y^{(\kappa+1)}\right)$ meets the power constraint (15) with equality. Since the total power consumption is monotonic in $\lambda$, the bisection search described in the Appendix can be used to find $\lambda$. Here, the initial values are chosen as $\lambda_{\mathrm{lo}}=0$ and

$$
\begin{aligned}
\lambda_{\text {hi }}=\max _{\substack{i=1, \ldots, M, \ell \in\{1,2\}}}\left\{\frac { 1 } { q _ { i } ^ { ( \ell ) } } \left[3 M \alpha_{i}^{(3-\ell)}(\kappa) / P\right.\right. \\
\left.-\left(\alpha_{i}^{(\ell)}(\kappa) d_{i}^{(\ell, \kappa)}+\sum_{t=1}^{2} \alpha_{i}^{(t)}(\kappa) a_{i} c_{i}^{(t, \kappa)}\right)\right], \\
\left.6 M p_{i}(\kappa) / P-r_{i}(\kappa)\right\} .
\end{aligned}
$$

In the case of the three separate power constraints (16), the solution of problem (34) s.t. (16) is derived as:

$$
x_{i}^{(\ell, \kappa+1)}=\frac{\alpha_{i}^{(3-\ell)}(\kappa)}{\alpha_{i}^{(\ell)}(\kappa) d_{i}^{(\ell, \kappa)}+\sum_{t=1}^{2} \alpha_{i}^{(t)}(\kappa) a_{i} c_{i}^{(t, \kappa)}+\lambda^{(\ell)} q_{i}^{(\ell)}}, \ell \in\{1,2\},
$$

$$
y_{i}^{(\kappa+1)}=\frac{2 p_{i}(\kappa)}{r_{i}(\kappa)+\lambda_{2}+\sqrt{\left(r_{i}(\kappa)+\lambda_{2}\right)^{2}+4 p_{i}(\kappa) q_{i}(\kappa)}},
$$

where $\lambda^{(\ell)}>0$ is chosen such that $x^{(\ell, \kappa+1)}$ meets the power constraint (16) with equality; $\lambda_{2}=0$ if $y^{(\kappa+1)}$ at $\lambda_{2}=0$ satisfies the power constraint (16), otherwise $\lambda_{2}>$ 0 is chosen such that $y^{(\kappa+1)}$ meets the power constraint (16) with equality. A bisection search similar to that in the Appendix can be used to find $\lambda^{(\ell)}, \ell \in\{1,2\}$ and $\lambda_{2}$, where the initial values are chosen as $\lambda_{\text {lo }}^{(\ell)}=\lambda_{2,1 o}=0$ and

$$
\begin{aligned}
\lambda_{\mathrm{hi}}^{(\ell)}=\max _{i=1, \ldots, M}\left\{\frac { 1 } { q _ { i } ^ { ( \ell ) } } \left[\frac{M \alpha_{i}^{(3-\ell)}(\kappa)}{P_{1}^{(\ell)}}-\left(\alpha_{i}^{(\ell)}(\kappa) d_{i}^{(\ell, \kappa)}\right.\right.\right. \\
\left.\left.\left.+\sum_{t=1}^{2} \alpha_{i}^{(t)}(\kappa) a_{i} c_{i}^{(t, \kappa)}\right)\right]\right\}, \ell \in\{1,2\}, \\
\lambda_{2, \mathrm{hi}}=\max _{i=1, \ldots, M}\left[2 M p_{i}(\kappa) / P_{2}-r_{i}(\kappa)\right] .
\end{aligned}
$$

The iterative waterfilling algorithm that solves problems (14) s.t. (15)/(16) is described as follows.

ALGORITHM 2. Initialized by a feasible solution $\left(x^{(1,0)}, x^{(2,0)}, y^{(0)}\right)$ to problems (14) s.t. $(15) /(16)$, generate a feasible solution $\left(x^{(1, \kappa+1)}, x^{(2, \kappa+1)}, y^{(\kappa+1)}\right)$ at $\kappa$-iteration for $\kappa=0,1, \ldots$, according to formulae (41)-(42)/(44)-(45).

Proposition 2 Initialized from a feasible solution $\left(x^{(1,0)}, x^{(2,0)}, y^{(0)}\right)$ to problems (14) s.t. (15)/(16), the sequence $\left\{\left(x^{(1, \kappa)}, x^{(2, \kappa)}, y^{(\kappa)}\right)\right\}$ generated by Algorithm 2 converges to at least a locally optimal solution of problems (14) s.t. $(15) /(16)$.

The HD two-way relaying problems (17) s.t. (15)/(16) are particular cases of the FD two-way relaying problems (14) s.t. (15)/(16) with $\gamma\left(\mathbf{x}_{i}^{(\ell)}, \mathbf{y}_{i}\right)=\mathbf{x}_{i}^{(\ell)}, \ell \in\{1,2\}$. Therefore, Algorithm 2 can be employed to solve the former by setting $\gamma \mathrm{LI}, i=0$ and using (17) to compute the achieved throughput.

Remark 1 The work of [27] addresses the HD twoway relaying problem (17) s.t. (15) by the following upper bound maximization:

$$
\max _{(\mathbf{x}, \mathbf{y})} \frac{1}{2} \sum_{i=1}^{M} \sum_{\ell=1}^{2} \ln \left(1+\frac{a_{i}\left(\mathbf{x}_{i}^{(1)}+\mathbf{x}_{i}^{(2)}\right) b_{i}^{(\ell)} \mathbf{y}_{i}}{a_{i}\left(\mathbf{x}_{i}^{(1)}+\mathbf{x}_{i}^{(2)}\right)+b_{i}^{(\ell)} \mathbf{y}_{i}}\right) \text { s.t. (15), }
$$

which is a convex but not necessarily computationally tractable problem. While the bound in (46) is not tight, applying the interior method to solve (46) as suggested in [27] is not computationally efficient.

\section{Tailored Solutions for One-Way Relaying}

Problems $(18) /(22)$ s.t. (19)/(20) are also particular cases of the problem (14) s.t. (15)/(16). However, their simpler structures entail more computationally efficient algorithms as will be developed in this section.

\subsection{FD One-Way Relaying}

As mentioned previously, the FD one-way relaying problems (18) s.t. (19)/(20) can be decomposed into two independent problems (21). Therefore, we solve problems (21) for each $\ell \in\{1,2\}$ here. The cross term $\gamma\left(\mathbf{x}_{i}^{(\ell)}, \mathbf{y}_{i}^{(\ell)}\right) \mathbf{y}_{i}^{(\ell)}$ in the objective function in (21) contributes greatly to the computational difficulty of these problems. Fortunately, we can separate these variables without any loss of accuracy. First, let us define:

$$
\begin{aligned}
f_{i}\left(\mathbf{x}_{i}^{(\ell)}, \mathbf{y}_{i}^{(\ell)}\right) \triangleq & \ln \left(1+a_{i}^{(\ell)} \mathbf{x}_{i}^{(\ell)}+\gamma \mathrm{Ll}, \mathbf{y}_{i}^{(\ell)}\right) \\
& +\ln \left(1+b_{i}^{(\ell)} \mathbf{y}_{i}^{(\ell)}\right) \\
g_{i}\left(\mathbf{x}_{i}^{(\ell)}, \mathbf{y}_{i}^{(\ell)}\right) \triangleq & \ln \left(1+a_{i}^{(\ell)} \mathbf{x}_{i}^{(\ell)}+\left(b_{i}^{(\ell)}+\gamma_{\mathrm{LL}, i}\right) \mathbf{y}_{i}^{(\ell)}\right. \\
& \left.+\gamma_{\mathrm{Ll}, i} b_{i}^{(\ell)}\left(\mathbf{y}_{i}^{(\ell)}\right)^{2}\right)
\end{aligned}
$$


for $i=1, \ldots, M$. We then rewrite the objective function in (21) as:

$$
\begin{gathered}
F\left(\mathbf{x}^{(\ell)}, \mathbf{y}^{(\ell)}\right)=\sum_{i=1}^{M}\left[\ln \left(1+a_{i}^{(\ell)} \gamma\left(\mathbf{x}_{i}^{(\ell)}, \mathbf{y}_{i}^{(\ell)}\right)\right)+\ln \left(1+b_{i}^{(\ell)} \mathbf{y}_{i}^{(\ell)}\right)\right. \\
\left.-\ln \left(1+a_{i}^{(\ell)} \gamma\left(\mathbf{x}_{i}^{(\ell)}, \mathbf{y}_{i}^{(\ell)}\right)+b_{i}^{(\ell)} \mathbf{y}_{i}^{(\ell)}\right)\right] \\
=\sum_{i=1}^{M}\left[\ln \left(1+a_{i}^{(\ell)} \mathbf{x}_{i}^{(\ell)}+\gamma_{\mathrm{LI}, i} \mathbf{y}_{i}^{(\ell)}\right)+\ln \left(1+b_{i}^{(\ell)} \mathbf{y}_{i}^{(\ell)}\right)\right. \\
-\ln \left(1+a_{i}^{(\ell)} \mathbf{x}_{i}^{(\ell)}+\left(b_{i}^{(\ell)}+\gamma \mathrm{LI}, i\right) \mathbf{y}_{i}^{(\ell)}\right. \\
\left.\left.+\gamma \mathrm{Ll}, i b_{i}^{(\ell)}\left(\mathbf{y}_{i}^{(\ell)}\right)^{2}\right)\right] \\
\triangleq f\left(\mathbf{x}^{(\ell)}, \mathbf{y}^{(\ell)}\right)-g\left(\mathbf{x}^{(\ell)}, \mathbf{y}^{(\ell)}\right),
\end{gathered}
$$

where the factor of $1 / 2$ is ignored without loss of optimality. Here,

$$
f\left(\mathbf{x}^{(\ell)}, \mathbf{y}^{(\ell)}\right) \triangleq \sum_{i=1}^{M} f_{i}\left(\mathbf{x}_{i}^{(\ell)}, \mathbf{y}_{i}^{(\ell)}\right)
$$

is concave, and

$$
g\left(\mathbf{x}^{(\ell)}, \mathbf{y}^{(\ell)}\right) \triangleq \sum_{i=1}^{M} g_{i}\left(\mathbf{x}_{i}^{(\ell)}, \mathbf{y}_{i}^{(\ell)}\right)
$$

is neither concave nor convex. By property (P1), we have that

$$
\begin{aligned}
& g_{i}\left(\mathbf{x}_{i}^{(\ell)}, \mathbf{y}_{i}^{(\ell)}\right) \\
& \leq g_{i}\left(x_{i}^{(\ell, \kappa)}, y_{i}^{(\ell, \kappa)}\right)+c_{i}^{(\ell, \kappa)}\left[\left(a_{i}^{(\ell)} \mathbf{x}_{i}^{(\ell)}+\left(b_{i}^{(\ell)}+\gamma \mathrm{LI}, i\right) \mathbf{y}_{i}^{(\ell)}\right.\right. \\
& \left.\quad+\gamma \mathrm{Ll}, i b_{i}^{(\ell)}\left(\mathbf{y}_{i}^{(\ell)}\right)^{2}\right)-\left(a_{i}^{(\ell)} x_{i}^{(\ell, \kappa)}+\left(b_{i}^{(\ell)}+\gamma \mathrm{LI}, i\right) y_{i}^{(\ell, \kappa)}\right. \\
& \left.\left.\quad+\gamma \mathrm{Ll}, i b_{i}^{(\ell)}\left(y_{i}^{(\ell, \kappa)}\right)^{2}\right)\right]
\end{aligned}
$$

for all $\mathbf{x}_{i}^{(\ell)} \geq 0, \mathbf{y}_{i}^{(\ell)} \geq 0, x_{i}^{(\ell, \kappa)} \geq 0, y_{i}^{(\ell, \kappa)} \geq 0$, where

$$
c_{i}^{(\ell, \kappa)}=\frac{1}{1+a_{i}^{(\ell)} x_{i}^{(\ell, \kappa)}+\left(b_{i}^{(\ell)}+\gamma_{\mathrm{LL}, i}\right) y_{i}^{(\ell, \kappa)}+\gamma_{\mathrm{LI}, i} b_{i}^{(\ell)}\left(y_{i}^{(\ell, \kappa)}\right)^{2}} .
$$

Then, the convex quadratic function $g^{(\kappa)}\left(\mathbf{x}^{(\ell)}, \mathbf{y}^{(\ell)}\right)$ defined by:

$$
\begin{aligned}
& g^{(\kappa)}\left(\mathbf{x}^{(\ell)}, \mathbf{y}^{(\ell)}\right) \\
& \triangleq g\left(x^{(\ell, \kappa)}, y^{(\ell, \kappa)}\right)+\sum_{i=1}^{M} c_{i}^{(\ell, \kappa)}\left(a_{i}^{(\ell)} \mathbf{x}_{i}^{(\ell)}+\left(b_{i}^{(\ell)}+\gamma_{\mathrm{L}, i}\right) \mathbf{y}_{i}^{(\ell)}+\gamma_{\mathrm{LL}, i} b_{i}^{(\ell)}\left(\mathbf{y}_{i}^{(\ell)}\right)^{2}\right) \\
& -\sum_{i=1}^{M} c_{i}^{(\ell, \kappa)}\left(a_{i}^{(\ell)} x_{i}^{(\ell, \kappa)}+\left(b_{i}^{(\ell)}+\gamma\llcorner\mathrm{L}, i) y_{i}^{(\ell, \kappa)}+\gamma \mathrm{LI}, i b_{i}^{(\ell)}\left(y_{i}^{(\ell, \kappa)}\right)^{2}\right)\right.
\end{aligned}
$$

provides the global upper bound $g\left(\mathbf{x}^{(\ell)}, \mathbf{y}^{(\ell)}\right)$ that matches $g(\cdot)$ at $\left(x^{(\ell, \kappa)}, y^{(\ell, \kappa)}\right)$, i.e.,

$$
\begin{aligned}
g\left(x^{(\ell, \kappa)}, y^{(\ell, \kappa)}\right) & =g^{(\kappa)}\left(x^{(\ell, \kappa)}, y^{(\ell, \kappa)}\right) \\
g\left(\mathbf{x}^{(\ell)}, \mathbf{y}^{(\ell)}\right) & \leq g^{(\kappa)}\left(\mathbf{x}^{(\ell)}, \mathbf{y}^{(\ell)}\right), \forall\left(\mathbf{x}^{(\ell)}, \mathbf{y}^{(\ell)}\right) .
\end{aligned}
$$

On the other hand, by property (P2), we have that

$$
\begin{aligned}
& \ln \left(1+a_{i}^{(\ell)} \mathbf{x}_{i}^{(\ell)}+\gamma \mathrm{LI}, \mathbf{y}_{i}^{(\ell)}\right) \\
& \geq \ln \left(1+a_{i}^{(\ell)} x_{i}^{(\ell, \kappa)}+\gamma \mathrm{LI}, y_{i}^{(\ell, \kappa)}\right)+p_{i}^{(\ell, \kappa)}\left(\ln \left(1+a_{i}^{(\ell)} \mathbf{x}_{i}^{(\ell)}\right)\right. \\
& \left.\quad-\ln \left(1+a_{i}^{(\ell)} x_{i}^{(\ell, \kappa)}\right)\right)+q_{i}^{(\ell, \kappa)}\left(\ln \mathbf{y}_{i}^{(\ell)}-\ln y_{i}^{(\ell, \kappa)}\right), \\
& \ln \left(1+b_{i}^{(\ell)} \mathbf{y}_{i}^{(\ell)}\right) \geq \ln \left(1+b_{i}^{(\ell)} y_{i}^{(\ell, \kappa)}\right)+r_{i}^{(\ell, \kappa)}\left(\ln \mathbf{y}_{i}^{(\ell)}-\ln y_{i}^{(\ell, \kappa)}\right),
\end{aligned}
$$

where

$$
\begin{aligned}
& p_{i}^{(\ell, \kappa)} \triangleq \frac{1+a_{i}^{(\ell)} x_{i}^{(\ell, \kappa)}}{1+a_{i}^{(\ell)} x_{i}^{(\ell, \kappa)}+\gamma_{\mathrm{LI}, i} y_{i}^{(\ell, \kappa)}}, \\
& q_{i}^{(\ell, \kappa)} \triangleq \frac{\gamma_{\mathrm{LI}, i} y_{i}^{(\ell, \kappa)}}{1+a_{i}^{(\ell)} x_{i}^{(\ell, \kappa)}+\gamma_{\mathrm{LI}, i} y_{i}^{(\ell, \kappa)}}, \\
& r_{i}^{(\ell, \kappa)} \triangleq \frac{b_{i}^{(\ell)} y_{i}^{(\ell, \kappa)}}{1+b_{i}^{(\ell)} y_{i}^{(\ell, \kappa)}} .
\end{aligned}
$$

As such, $f\left(\mathbf{x}^{(\ell)}, \mathbf{y}^{(\ell)}\right) \geq f^{(\kappa)}\left(\mathbf{x}^{(\ell)}, \mathbf{y}^{(\ell)}\right)$ and $f\left(x^{(\ell, \kappa)}\right.$, $\left.y^{(\ell, \kappa)}\right)=f^{(\kappa)}\left(x^{(\ell, \kappa)}, y^{(\ell, \kappa)}\right)$ for

$$
\begin{aligned}
& f^{(\kappa)}\left(\mathbf{x}^{(\ell)}, \mathbf{y}^{(\ell)}\right) \\
& \triangleq f\left(x^{(\ell, \kappa)}, y^{(\ell, \kappa)}\right)+\sum_{i=1}^{M}\left[p _ { i } ^ { ( \ell , \kappa ) } \left(\ln \left(1+a_{i}^{(\ell)} \mathbf{x}_{i}^{(\ell)}\right)\right.\right. \\
& \left.\quad-\ln \left(1+a_{i}^{(\ell)} x_{i}^{(\ell, \kappa)}\right)\right)+q_{i}^{(\ell, \kappa)}\left(\ln \mathbf{y}_{i}^{(\ell)}-\ln y_{i}^{(\ell, \kappa)}\right) \\
& \left.\quad+r_{i}^{(\ell, \kappa)}\left(\ln \mathbf{y}_{i}^{(\ell)}-\ln y_{i}^{(\ell, \kappa)}\right)\right] .
\end{aligned}
$$

Therefore, the function $F^{(\kappa)} \triangleq f^{(\kappa)}\left(\mathbf{x}^{(\ell)}, \mathbf{y}^{(\ell)}\right)-$ $g^{(\kappa)}\left(\mathbf{x}^{(\ell)}, \mathbf{y}^{(\ell)}\right)$ satisfies the two crucial properties (27) and (28) for the applicability of Algorithm 1. The corresponding problem (29) boils down to the following convex program:

$$
\begin{aligned}
& \max _{\left(\mathbf{x}^{(\ell)}, \mathbf{y}^{(\ell)}\right)} \sum_{i=1}^{M}[ p_{i}^{(\ell, \kappa)} \ln \left(1+a_{i}^{(\ell)} \mathbf{x}_{i}^{(\ell)}\right)+\left(q_{i}^{(\ell, \kappa)}+r_{i}^{(\ell, \kappa)}\right) \ln \mathbf{y}_{i}^{(\ell)} \\
& \quad-c_{i}^{(\ell, \kappa)}\left(a_{i}^{(\ell)} \mathbf{x}_{i}^{(\ell)}+\left(b_{i}^{(\ell)}+\gamma \mathrm{LL}, i\right) \mathbf{y}_{i}^{(\ell)}+\gamma \mathrm{LL}, i\right. \\
&\left.\left.b_{i}^{(\ell)}\left(\mathbf{y}_{i}^{(\ell)}\right)^{2}\right)\right]
\end{aligned}
$$

s.t. $(19) /(20)$. 
In the case of constraint (19), the KKT conditions for necessary and sufficient optimality are

$$
\begin{array}{r}
\frac{a_{i}^{(\ell)} p_{i}^{(\ell, \kappa)}}{1+a_{i}^{(\ell)} \mathbf{x}_{i}^{(\ell)}}-c_{i}^{(\ell, \kappa)} a_{i}^{(\ell)}+\lambda_{1 i}=\lambda, \\
\lambda\left(\sum_{i=1}^{M}\left(\mathbf{x}_{i}^{(\ell)}+\mathbf{y}_{i}^{(\ell)}\right)-P / 2\right)=0, \\
\frac{q_{i}^{(\ell, \kappa)}+r_{i}^{(\ell, \kappa)}}{\mathbf{y}_{i}^{(\ell)}}-c_{i}^{(\ell, \kappa)}\left[\left(b_{i}^{(\ell)}+\gamma_{\mathrm{L}, i}\right)+2 \gamma_{\mathrm{L}, i} b_{i}^{(\ell)} \mathbf{y}_{i}^{(\ell)}\right]+\lambda_{2 i}=\lambda, \\
\lambda_{1 i} \mathbf{x}_{i}^{(\ell)}=0, \lambda_{2 i} \mathbf{y}_{i}^{(\ell)}=0, \lambda_{1 i} \geq 0, \lambda_{2 i} \geq 0, \lambda \geq 0
\end{array}
$$

for $i=1, \ldots, M$. The optimal solution $\left(x^{(\ell, \kappa+1)}, y^{(\ell, \kappa+1)}\right)$ of problem (29) s.t. (19) is then derived as:

$$
\begin{aligned}
x_{i}^{(\ell, \kappa+1)}= & \max \left\{\frac{p_{i}^{(\ell, \kappa)}}{c_{i}^{(\ell, \kappa)} a_{i}^{(\ell)}+\lambda}-\frac{1}{a_{i}^{(\ell)}}, 0\right\}, \\
y_{i}^{(\ell, \kappa+1)}= & 2\left(q_{i}^{(\ell, \kappa)}+r_{i}^{(\ell, \kappa)}\right)\left[c_{i}^{(\ell, \kappa)}\left(b_{i}^{(\ell)}+\gamma_{\mathrm{L}, i}\right)+\lambda\right. \\
& \left.+\sqrt{\left(c_{i}^{(\ell, \kappa)}\left(b_{i}^{(\ell)}+\gamma \mathrm{LL}, i\right)+\lambda\right)^{2}+8\left(q_{i}^{(\ell, \kappa)}+r_{i}^{(\ell, \kappa)}\right) c_{i}^{(\ell, \kappa)} \gamma_{L, i} b_{i}^{(\ell)}}\right]^{-1},
\end{aligned}
$$

where $\lambda>0$ is chosen such that $\left(x^{(\ell, \kappa+1)}, y^{(\ell, \kappa+1)}\right)$ meets the power constraint (19) with equality. A bisection search similar to that in the Appendix can be used, where the initial values are set as $\lambda_{\mathrm{lo}}=0$ and

$$
\begin{aligned}
\lambda_{\mathrm{hi}}=\max _{i=1, \ldots, M}\left\{\frac{p_{i}^{(\ell, \kappa)}}{P /(6 M)+1 / a_{i}^{(\ell)}}-c_{i}^{(\ell, \kappa)} a_{i}^{(\ell)},\right. \\
\left.\frac{12 M}{P}\left(q_{i}^{(\ell, \kappa)}+r_{i}^{(\ell, \kappa)}\right)-c_{i}^{(\ell, \kappa)}\left(b_{i}^{(\ell)}+\gamma_{\mathrm{LL}, i}\right)\right\} .
\end{aligned}
$$

Analogously, in the case of constraints (20), the optimal solution of problem (29) s.t. (20) is derived as:

$$
\begin{aligned}
x_{i}^{(\ell, \kappa+1)} & =\max \left\{\frac{p_{i}^{(\ell, \kappa)}}{c_{i}^{(\ell, \kappa)} a_{i}^{(\ell)}+\lambda_{1}^{(\ell)}}-\frac{1}{a_{i}^{(\ell)}}, 0\right\}, \\
y_{i}^{(\ell, \kappa+1)}= & 2\left(q_{i}^{(\ell, \kappa)}+r_{i}^{(\ell, \kappa)}\right)\left[c_{i}^{(\ell, \kappa)}\left(b_{i}^{(\ell)}+\gamma_{\llcorner, i}\right)+\lambda_{2}^{(\ell)}\right. \\
& \left.+\sqrt{\left(c_{i}^{(\ell, \kappa)}\left(b_{i}^{(\ell)}+\gamma_{L, i}\right)+\lambda_{2}^{(\ell)}\right)^{2}+8\left(q_{i}^{(\ell, \kappa)}+r_{i}^{(\ell, \kappa)}\right) c_{i}^{(\ell, \kappa)} \eta_{\mathrm{L}, i} b_{i}^{(\ell)}}\right]^{-1}
\end{aligned}
$$

where $\lambda_{1}^{(\ell)}>0$ is chosen such that $\sum_{i=1}^{M} x_{i}^{(\ell, \kappa+1)}=P_{1}^{(\ell)}$; $\lambda_{2}^{(\ell)}=0$ if $\sum_{i=1}^{M} y_{i}^{(\ell, \kappa+1)} \leq P_{2} / 2$ at $\lambda_{2}^{(\ell)}=0$, otherwise $\lambda_{2}^{(\ell)}>0$ is chosen such that $\sum_{i=1}^{M} y_{i}^{(\ell, \kappa+1)}=P_{2} / 2$. A bisection search similar to that in the Appendix can be used, where the initial values are set as $\lambda_{1, \mathrm{lo}}^{(\ell)}=\lambda_{2, \mathrm{lo}}^{(\ell)}=0$ and

$$
\begin{aligned}
& \lambda_{1, \mathrm{hi}}^{(\ell)}=\max _{i=1, \ldots, M}\left\{\frac{p_{i}^{(\ell, \kappa)}}{P_{1}^{(\ell)} / M+1 / a_{i}^{(\ell)}}-c_{i}^{(\ell, \kappa)} a_{i}^{(\ell)}\right\}, \\
& \lambda_{2, \mathrm{hi}}^{(\ell)}=\max _{i=1, \ldots, M}\left\{\frac{4 M}{P_{2}}\left(q_{i}^{(\ell, \kappa)}+r_{i}^{(\ell, \kappa)}\right)-c_{i}^{(\ell, \kappa)}\left(b_{i}^{(\ell)}+\gamma \mathrm{LL}, i\right)\right\} .
\end{aligned}
$$

The iterative waterfilling algorithm that solves problems (18) s.t. $(19) /(20)$ is described as follows.

ALGORITHM 3. Initialized by a feasible solution $\left(x^{(\ell, 0)}, y^{(\ell, 0)}\right), \ell \in\{1,2\}$ to problems (18) s.t. $(19) /(20)$, generate a feasible solution $\left(x^{(\ell, \kappa+1)}, y^{(\ell, \kappa+1)}\right)$ at $\kappa$-iteration for $\kappa=0,1, \ldots$, according to formulae (60)-(61)/(62)-(63).

Proposition 3 Initialized from a feasible solution $\left(x^{(\ell, 0)}, y^{(\ell, 0)}\right), \ell \in\{1,2\}$ to problems (18) s.t. $(19) /(20)$, the sequence $\left\{\left(x^{(\ell, \kappa)}, y^{(\ell, \kappa)}\right)\right\}$ of improved solutions generated by Algorithm 3 converges to at least a locally optimal solution of problems (18) s.t. (19)/(20).

\subsection{HD One-Way Relaying}

The HD one-way relaying problems (22) s.t. (19)/(20) correspond to the FD one-way relaying problems (18) s.t. (19)/(20) with $\gamma \mathrm{LI}, i=0$. Therefore, Algorithm 3 can be employed to solve the former by simply setting $\gamma_{\mathrm{LI}, i}=0$ and using (22) to compute the achieved throughput. In this special case, we can also derive even simpler waterfilling solutions for these problems as follows. First note that $f^{(\kappa)}\left(\mathbf{x}^{(\ell)}, \mathbf{y}^{(\ell)}\right) \equiv f\left(\mathbf{x}^{(\ell)}, \mathbf{y}^{(\ell)}\right)$, so we do not need to approximate $f\left(\mathbf{x}^{(\ell)}, \mathbf{y}^{(\ell)}\right)$ and problem (29) then admits a closed-form optimal solution.

For the joint sum-power constraint (19), the optimal solution for problem (22) s.t. (19) is derived as:

$$
\begin{aligned}
& x_{i}^{(\ell, \kappa+1)}=\max \left\{\frac{1}{c_{i}^{(\ell, \kappa)} a_{i}^{(\ell)}+\lambda}-\frac{1}{a_{i}^{(\ell)}}, 0\right\} \\
& y_{i}^{(\ell, \kappa+1)}=\max \left\{\frac{1}{c_{i}^{(\ell, \kappa)} b_{i}^{(\ell)}+\lambda}-\frac{1}{b_{i}^{(\ell)}}, 0\right\},
\end{aligned}
$$

where $\lambda>0$ is chosen such that the constraint (19) is met with equality for $\left(x^{(\ell, \kappa+1)}, y^{(\ell, \kappa+1)}\right)$. A bisection search similar to that in the Appendix can be used, where the initial values are set as $\lambda_{\mathrm{lo}}=0$ and

$$
\begin{aligned}
\lambda_{\mathrm{hi}}=\max _{i=1, \ldots, M} & \left\{\frac{1}{P /(6 M)+1 / a_{i}^{(\ell)}}-c_{i}^{(\ell, \kappa)} a_{i}^{(\ell)},\right. \\
& \left.\frac{1}{P /(6 M)+1 / b_{i}^{(\ell)}}-c_{i}^{(\ell, \kappa)} b_{i}^{(\ell)}\right\} .
\end{aligned}
$$


Analogously, for the separate sum-power constraints (20), the optimal solution for problem (22) s.t. (20) is derived as:

$$
\begin{aligned}
& x_{i}^{(\ell, \kappa+1)}=\max \left\{\frac{1}{c_{i}^{(\ell, \kappa)} a_{i}^{(\ell)}+\lambda_{1}^{(\ell)}}-\frac{1}{a_{i}^{(\ell)}}, 0\right\}, \\
& y_{i}^{(\ell, \kappa+1)}=\max \left\{\frac{1}{c_{i}^{(\ell, \kappa)} b_{i}^{(\ell)}+\lambda_{2}^{(\ell)}}-\frac{1}{b_{i}^{(\ell)}}, 0\right\} \text {, }
\end{aligned}
$$

where $\lambda_{1}^{(\ell)}>0$ and $\lambda_{2}^{(\ell)}>0$ are chosen such that the constraints (20) are met with equality for $\left(x^{(\ell, \kappa+1)}, y^{(\ell, \kappa+1)}\right)$. A bisection search similar to that in the Appendix can be used, where the initial values are set as $\lambda_{1, \mathrm{lo}}^{(\ell)}=\lambda_{2, \mathrm{lo}}^{(\ell)}=0$ and $\lambda_{1, \mathrm{hi}}^{(\ell)}=$ $\max _{i=1, \ldots, M}\left[1 /\left(P_{1}^{(\ell)} / M+1 / a_{i}^{(\ell)}\right)-c_{i}^{(\ell, \kappa)} a_{i}^{(\ell)}\right], \lambda_{2, \mathrm{hi}}^{(\ell)}=$ $\max _{i=1, \ldots, M}\left[1 /\left(P_{2} /(2 M)+b_{i}^{(\ell)}\right)-c_{i}^{(\ell, \kappa)} b_{i}^{(\ell)}\right]$.

The iterative waterfilling algorithm that solves problems (22) s.t. (19)/(20) is described as follows.

ALGORITHM 4. Initialized by a feasible solution $\left(x^{(\ell, 0)}, y^{(\ell, 0)}\right), \ell \in\{1,2\}$ to problems (22) s.t. (19)/(20), generate a feasible solution $\left(x^{(\ell, \kappa+1)}, y^{(\ell, \kappa+1)}\right)$ at $\kappa$-iteration for $\kappa=0,1, \ldots$, according to formulae (64)/(62)-(65).

Remark 2 The work of [16] addresses problem (22) s.t. (19) by using the following approximation:

$$
\frac{a_{i}^{(\ell)} \mathbf{x}_{i}^{(\ell)} b_{i}^{(\ell)} \mathbf{y}_{i}^{(\ell)}}{1+a_{i}^{(\ell)} \mathbf{x}_{i}^{(\ell)}+b_{i}^{(\ell)} \mathbf{y}_{i}^{(\ell)}} \approx \frac{a_{i}^{(\ell)} \mathbf{x}_{i}^{(\ell)} b_{i}^{(\ell)} \mathbf{y}_{i}^{(\ell)}}{a_{i}^{(\ell)} \mathbf{x}_{i}^{(\ell)}+b_{i}^{(\ell)} \mathbf{y}_{i}^{(\ell)}}, \ell \in\{1,2\}
$$

which is only valid for high SNR values in subcarrier $i$. As mentioned before, this SNR assumption does not always hold for OFDM systems. With (66), [16] considers the following problem instead of problem (22) s.t. (19):

$$
\max _{\left(\mathbf{x}^{(\ell)}, \mathbf{y}^{(\ell)}\right)} \sum_{i=1}^{M} \ln \left(1+\frac{a_{i}^{(\ell)} \mathbf{x}_{i}^{(\ell)} b_{i}^{(\ell)} \mathbf{y}_{i}^{(\ell)}}{a_{i}^{(\ell)} \mathbf{x}_{i}^{(\ell)}+b_{i}^{(\ell)} \mathbf{y}_{i}^{(\ell)}}\right)
$$

It is seen that $\max _{\mathbf{x}_{i}^{(\ell)}+\mathbf{y}_{i}^{(\ell)} \triangleq \mathbf{z}_{i}^{(\ell)}} \frac{a_{i}^{(\ell)} \mathbf{x}_{i}^{(\ell)} b_{i}^{(\ell)} \mathbf{y}_{i}^{(\ell)}}{a_{i}^{(\ell)} \mathbf{x}_{i}^{(\ell)}+b_{i}^{(\ell)} \mathbf{y}_{i}^{(\ell)}}=a_{i}^{(\ell)} b_{i}^{(\ell)}$ $\frac{\eta_{i}^{(\ell)}\left(1-\eta_{i}^{(\ell)}\right)}{a_{i}^{(\ell)} \eta_{i}^{(\ell)}+\left(1-\eta_{i}^{(\ell)}\right) b_{i}^{(\ell)}} \mathbf{z}_{i}^{(\ell)}$ for $\eta_{i}^{(\ell)}=\frac{b_{i}^{(\ell)}-\sqrt{a_{i}^{(\ell)} b_{i}^{(\ell)}}}{b_{i}^{(\ell)}-a_{i}^{(\ell)}} ; 1-\eta_{i}^{(\ell)}=$ $\frac{a_{i}^{(\ell)}-\sqrt{a_{i}^{(\ell)} b_{i}^{(\ell)}}}{a_{i}^{(\ell)}-b_{i}^{(\ell)}}$ which is attained at

$$
\mathbf{x}_{i}^{(\ell)}=\eta_{i}^{(\ell)} \mathbf{z}_{i}^{(\ell)}, \quad \mathbf{y}_{i}^{(\ell)}=\left(1-\eta_{i}^{(\ell)}\right) \mathbf{z}_{i}^{(\ell)}
$$

Thus, (67) is equivalent to the following convex problem:

$$
\begin{aligned}
\underset{\substack{\mathbf{z}_{(\ell)}^{(\ell)} \triangleq \\
\left(\mathbf{z}_{1}^{(\ell)}, \ldots, \mathbf{z}_{M}^{(\ell)}\right)^{T}}}{ } & \sum_{i=1}^{M} \ln \left(1+a_{i}^{(\ell)} b_{i}^{(\ell)} \frac{\eta_{i}^{(\ell)}\left(1-\eta_{i}^{(\ell)}\right)}{a_{i}^{(\ell)} \eta_{i}^{(\ell)}+\left(1-\eta_{i}^{(\ell)}\right) b_{i}^{(\ell)}} \mathbf{z}_{i}^{(\ell)}\right) \\
\text { s.t. } & \sum_{i=1}^{M} \mathbf{z}_{i}^{(\ell)} \leq P / 2,
\end{aligned}
$$

which has a closed-form optimal solution [16, (12)-(13)]. As

$$
\frac{a_{i}^{(\ell)} \mathbf{x}_{i}^{(\ell)} b_{i}^{(\ell)} \mathbf{y}_{i}^{(\ell)}}{1+a_{i}^{(\ell)} \mathbf{x}_{i}^{(\ell)}+b_{i}^{(\ell)} \mathbf{y}_{i}^{(\ell)}}<\frac{a_{i}^{(\ell)} \mathbf{x}_{i}^{(\ell)} b_{i}^{(\ell)} \mathbf{y}_{i}^{(\ell)}}{a_{i}^{(\ell)} \mathbf{x}_{i}^{(\ell)}+b_{i}^{(\ell)} \mathbf{y}_{i}^{(\ell)}},
$$

problem (67) is in fact an upper bound maximization of problem (22) s.t. (19). However, it should be noted that a natural approximated optimization of the maximization problem (22) s.t. (19) should always be a lower bound maximization.

Also, the work of [17] addresses the joint sum-power constrained problem (18) s.t. (19) by assuming high SNR scenarios. Using the approximations similar to [16], i.e.,

$$
\begin{aligned}
& \gamma_{i}\left(\mathbf{x}_{i}^{(\ell)}, \mathbf{y}_{i}^{(\ell)}\right)=\frac{\mathbf{x}_{i}^{(\ell)}}{\gamma \mathrm{Ll}, \mathbf{y}_{i}^{(\ell)}+1} \approx \frac{\mathbf{x}_{i}^{(\ell)}}{\gamma \mathrm{Ll}, i \mathbf{y}_{i}^{(\ell)}}, \quad(70) \\
& \frac{a_{i}^{(\ell)} \gamma\left(\mathbf{x}_{i}^{(\ell)}, \mathbf{y}_{i}^{(\ell)}\right) b_{i}^{(\ell)} \mathbf{y}_{i}^{(\ell)}}{1+a_{i}^{(\ell)} \gamma\left(\mathbf{x}_{i}^{(\ell)}, \mathbf{y}_{i}^{(\ell)}\right)+b_{i}^{(\ell)} \mathbf{y}_{i}^{(\ell)}} \approx \frac{a_{i}^{(\ell)} \gamma\left(\mathbf{x}_{i}^{(\ell)}, \mathbf{y}_{i}^{(\ell)}\right) b_{i}^{(\ell)} \mathbf{y}_{i}^{(\ell)}}{a_{i}^{(\ell)} \gamma\left(\mathbf{x}_{i}^{(\ell)}, \mathbf{y}_{i}^{(\ell)}\right)+b_{i}^{(\ell)} \mathbf{y}_{i}^{(\ell)}} \\
& \approx \frac{a_{i}^{(\ell)} \frac{\mathbf{x}_{i}^{(\ell)}}{\gamma_{\mathrm{LI}, i} \mathbf{y}_{i}^{(\ell)}} b_{i}^{(\ell)} \mathbf{y}_{i}^{(\ell)}}{a_{i}^{(\ell)} \frac{\mathbf{x}_{i}^{(\ell)}}{\gamma \mathrm{L}, i \mathbf{y}_{i}^{(\ell)}}+b_{i}^{(\ell)} \mathbf{y}_{i}^{(\ell)}}
\end{aligned}
$$$$
\begin{aligned}
& \approx \frac{\left(\mathbf{x}_{i}^{(\ell)}+\mathbf{y}_{i}^{(\ell)}\right)^{2} a_{i}^{(\ell)} b_{i}^{(\ell)}}{a_{i}^{(\ell)}\left(\mathbf{x}_{i}^{(\ell)}+\mathbf{y}_{i}^{(\ell)}\right)+2 \sqrt{\gamma \mathrm{Ll}, i} a_{i}^{(\ell)} b_{i}^{(\ell)}\left(\mathbf{x}_{i}^{(\ell)}+\mathbf{y}_{i}^{(\ell)}\right)^{3}} \\
& \approx \sqrt{a_{i}^{(\ell)} b_{i}^{(\ell)}\left(\mathbf{x}_{i}^{(\ell)}+\mathbf{y}_{i}^{(\ell)}\right)} /(2 \sqrt{\gamma \mathrm{LI}, i}),
\end{aligned}
$$

the following convex relaxation is considered in [17] instead of problem (18) s.t. (19):

$$
\begin{aligned}
& \max _{\mathbf{z}^{(\ell)} \triangleq\left(\mathbf{z}_{1}^{(\ell)}, \ldots, \mathbf{z}_{M}^{(\ell)}\right)^{T}} \sum_{i=1}^{M} \ln \left(1+\frac{\sqrt{a_{i}^{(\ell)} b_{i}^{(\ell)}}}{2 \sqrt{\gamma \mathrm{LI}, i}} \sqrt{\mathbf{z}_{i}^{(\ell)}}\right) \\
& \text { s.t. } \sum_{i=1}^{M} \mathbf{z}_{i}^{(\ell)} \leq P / 2 \text {, }
\end{aligned}
$$

for which a closed-form expression of the optimal solution can be obtained. One could see that the approximation (70) is poor for small values of $\gamma \mathrm{LI}, i$ (e.g., in FD relays with effective self-interference cancelation). Likewise, because 


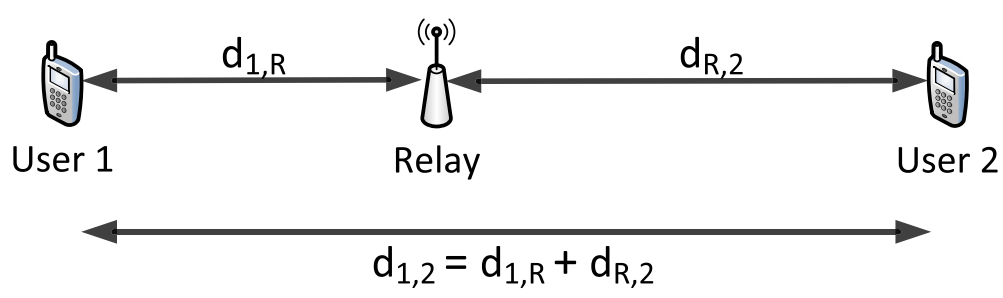

Fig. 2 The relaying network model used in the simulations

(71) ignores the essential term $a_{i}^{(\ell)}\left(\mathbf{x}_{i}^{(\ell)}+\mathbf{y}_{i}^{(\ell)}\right)$ in the denominator, this approximation is hardly valid for large values of $a_{i}^{(\ell)}\left(\mathbf{x}_{i}^{(\ell)}+\mathbf{y}_{i}^{(\ell)}\right)$. As more power is allocated to the good OFDM subcarriers to maximize the throughput, it is reasonable to expect a large value of $a_{i}^{(\ell)}\left(\mathbf{x}_{i}^{(\ell)}+\mathbf{y}_{i}^{(\ell)}\right)$ for some subcarrier i. Again (72) is upper bound maximization for the maximization problem (18) s.t. (19) while its lower bound maximization is always desirable. This kind of convex relaxation for the joint sum-power constraint is not applicable to the case of separate sum-power constraints in $\mathbf{x}_{i}^{(\ell)}$ and $\mathbf{y}_{i}^{(\ell)}$ because the power distribution (68) does not hold in the latter case.

\section{Numerical Results}

We consider a two-hop relaying network depicted in Fig. 2. Since the two user nodes and the relay are collocated on a line, $d_{1,2}=d_{1, R}+d_{R, 2}$. In all simulations, we set the number of antennas as $N=4$. For each spatial channel, we use the following pathloss model [31]:

$$
P L_{d B}=38+30 \log _{10}(d)+\psi,
$$

where $d$ (in meters) is the transmitter-receiver distance and $\psi$ (in $\mathrm{dB}$ ) is a correction factor (e.g., to model the outdoor wall penetration loss). We model the effect of shadowing by a log-normal random variable with mean of zero and standard deviation of $6 \mathrm{~dB}$. To simulate the effect of frequency selectivity in each spatial channel, we assume an exponential power delay profile (PDP) with a rootmean-square (RMS) delay spread of $\sigma_{\mathrm{RMS}}=3 T_{s}$ where $T_{s}$ is a constant. We model the magnitude of the timedomain channel corresponding to each tap of the PDP by either Rayleigh or Rician distribution. We further model the spatial correlation among the MIMO channels according to Case B of the 3GPP I-METRA MIMO channel model ([32], p.94).

The time-domain channels are converted to the frequency domain by the Fast Fourier transform (FFT) for the computation of the OFDM throughput. We use $K=1,024$ OFDM subcarriers, each of which occupies a bandwidth of $\Delta f=15 \mathrm{kHz}$. Since we take $T_{s}=$ $1 /(K \Delta f), \Delta f$ is much smaller than the channel coherence bandwidth of $0.02 / \sigma_{\text {RMS }}$ ([33], p.85). The OFDM subchannels are frequency-flat while there is correlation

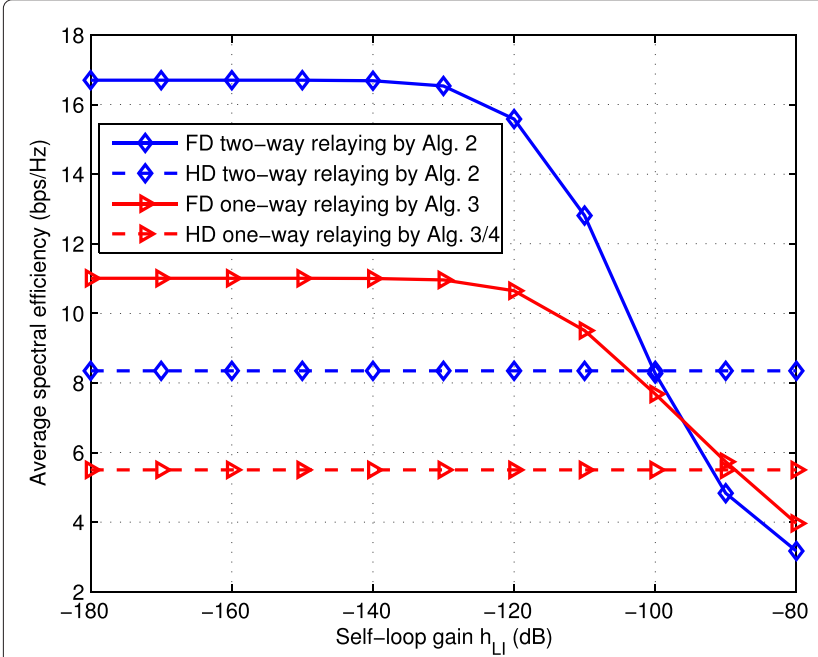

(a)

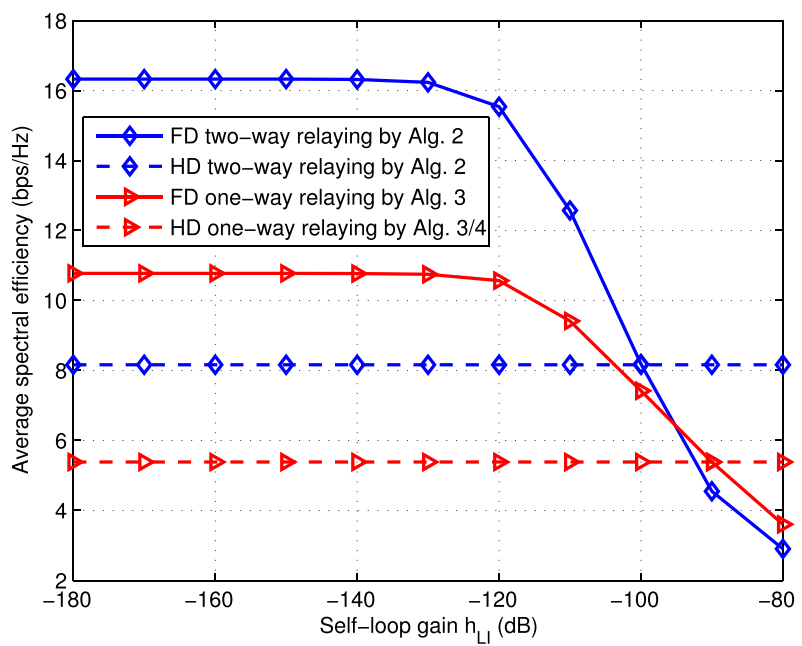

(b)

Fig. 3 Average spectral efficiency in the D2D communication with UE relaying scenario. a Joint sum-power constraint with $P_{2}=20 \mathrm{dBm}$. b Separate sum-power constraints with $P_{2}=20 \mathrm{dBm}$ 
among the adjacent subchannels. In each subchannel, the power spectral density of additive white Gaussian background noise at each antenna is $-174 \mathrm{dBm} / \mathrm{Hz}$, and the correlation between noise samples from different antennas is 0.2. The effect of all other impairments (including inter-carrier power leakage) is modelled as additive Gaussian noise whose power is twice that of the background noise. We set the error tolerance for all algorithms as $\epsilon=$ $10^{-4}$. We repeat the simulation for 100 independent runs and average the results to get the final figures for spectral efficiency.

We evaluate the performance of $\mathrm{FD} / \mathrm{HD}$ two-way relaying (by Algorithm 2) and FD one-way relaying (by Algorithm 3). We use the HD one-way relaying result (by
Algorithms 3 and 4) as the baseline performance. For a fair comparison with two-way relaying, we average the throughput of the one-way relaying scheme in two different directions, i.e., one from user 1 to relay to user 2, and another from user 2 to relay to user 1 . We set the maximum transmit power at the relay as $P_{2}$ in the twoway relaying and as $P_{2} / 2$ in each direction of the one-way relaying. We assume that $P_{1}^{(1)}+P_{2} / 2=P_{2}^{(1)}+P_{2} / 2=P / 2$. For simplicity, we set the instantaneous self-loop gain as $h_{\mathrm{Ll}, k, n}=h_{\mathrm{LL}, k, n}^{S} \equiv h_{\mathrm{LI}}$. Note that the presented value of $h_{\mathrm{LI}}$ is not normalized with respect to noise power, and that $h_{\mathrm{LI}}=0$ in the HD two-way/one-way relaying. After normalization, the values of $h_{\mathrm{LI}}$ would be consistent with those used in the literature, e.g., [17]. In all the results,

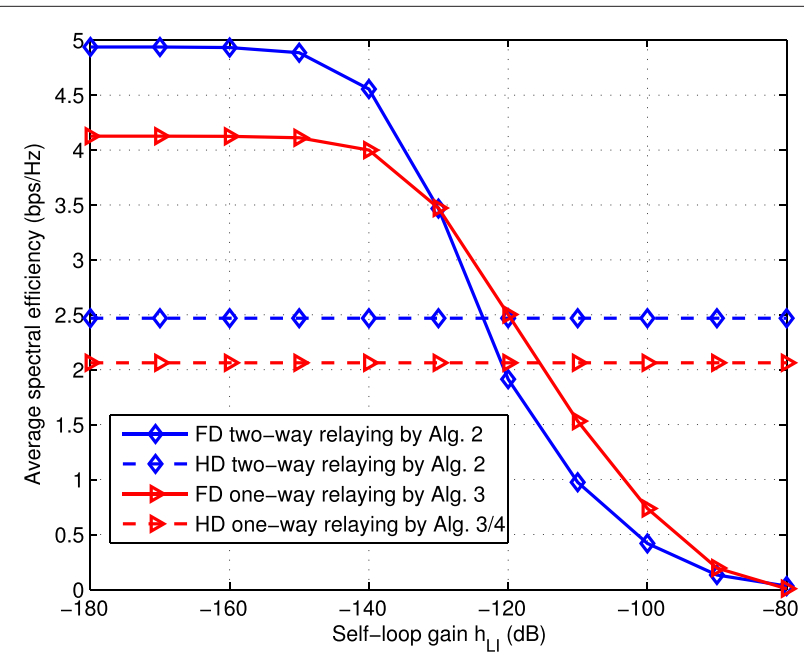

(a)

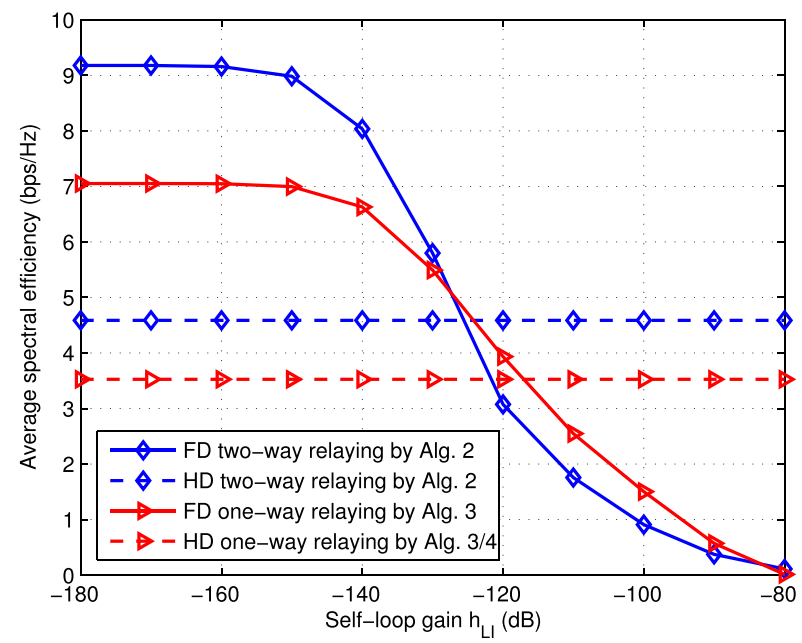

(c)

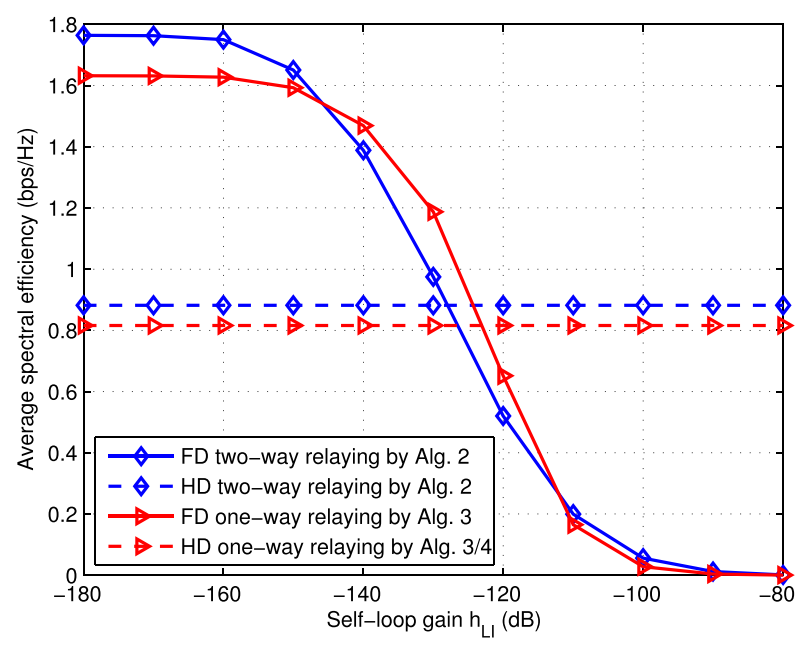

(b)

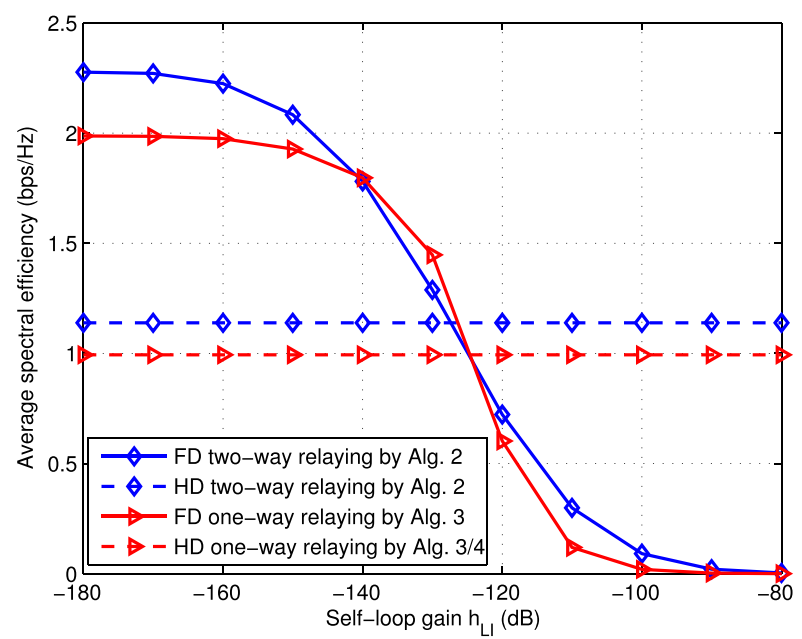

(d)

Fig. 4 Average spectral efficiency in the UE-UE communication with BS relaying scenario. a Joint sum-power constraint with $P_{2}=40 \mathrm{dBm}$. b Separate sum-power constraints with $P_{2}=40 \mathrm{dBm}$. c Joint sum-power constraint with $P_{2}=46 \mathrm{dBm}$. d Separate sum-power constraints with $P_{2}=46 \mathrm{dBm}$ 
we initialize Algorithm 2 by the feasible points $x_{i}^{(\ell)}=$ $P_{1}^{(\ell)} /\left(M \max _{i} q_{i}^{(\ell)}\right), \ell \in\{1,2\}$ and $y_{i}=P_{2} / M$ for all $i=1, \ldots, M$ (where $M=N K=4,096$ ). And we initialize Algorithms 3 and 4 by $x_{i}^{(\ell)}=P_{1}^{(\ell)} / M$ and $y_{i}=P_{2} / M$ for $\ell \in\{1,2\}$ and $i=1, \ldots, M$.

In the first example, we set $d_{1, R}=d_{R, 2}=50 \mathrm{~m}, \psi=$ OdB, $P_{1}^{(1)}=P_{1}^{(2)}=P_{2}=20 \mathrm{dBm}$. Assume there is a line of sight between a user and the relay. Each tap of the PDP from a user to the relay follows the Rician distribution with parameter $K_{r}=1$. This setup resembles the scenario of device-to-device (D2D) communication with UE relaying. From Fig. 3, the achieved throughput by FD almost doubles that by HD at the low values of $h_{\mathrm{LI}}$ in both one-way and two-way relaying cases. However, the throughput declines as $h_{\mathrm{LI}}$ increases, confirming the intuition that the self-loop interference (either at the user terminals or the relay) is the limiting parameter for FD transmissions to be beneficial. Particularly, the gain provided by $\mathrm{FD}$ vanishes beyond $h_{\mathrm{LI}}=-100 \mathrm{~dB}$, i.e., it benefits more to stay with the HD transmission beyond this point. Since the throughput by Algorithm 4 is almost identical to that by Algorithm 3, we only present one curve for the HD one-way relaying result to keep Fig. 3 clear.

Both HD two-way relaying and HD one-way relaying are not subjected to any SI. However, Fig. 3 shows that HD two-way relaying does not double the throughput of $\mathrm{HD}$ one-way relaying. This is because in the two-way relaying, (i) our precoding design may have amplified noise, (ii) the available power is further constrained by (15) and (16), and (iii) the denominator of the SINR expression is $a_{i}\left(\mathbf{x}_{i}^{(1)}+\mathbf{x}_{i}^{(2)}\right)$ which is greater than or equal to $a_{i}^{(\ell)} \mathbf{x}_{i}^{(\ell)}, \ell \in\{1,2\}$ of the one-way relaying. The gain from using half the number of time slots by two-way relaying may not be sufficient to offset the loss in the achieved throughput in each way. Similar reasons apply to FD two-way relaying where this strategy is further subjected to the SI at the users. Therefore, although FD two-relaying uses only one time slot for transmissions in both link directions, it does not double the throughput of FD one-way relaying. When SI is large (i.e., $h_{\mathrm{LI}} \geq-100 \mathrm{~dB}$ ), Fig. 3 reveals that FD twoway relaying is even slightly outperformed by FD one-way relaying.

In the second example, we set $d_{1, R}=d_{R, 2}=500 \mathrm{~m}, \psi=$ OdB, $P_{1}^{(1)}=P_{1}^{(2)}=20 \mathrm{dBm}$, and $P_{2}$ to be either $40 \mathrm{dBm}$ or $46 \mathrm{dBm}$. We assume there is no line of sight between each user and the relay, so each tap of the corresponding channel PDP follows the Rayleigh distribution. This setup simulates the scenario of UE-UE communication with BS relaying. As the power budget respectively increases by $20 \mathrm{~dB}$ and $26 \mathrm{~dB}$ compared to that shown in Fig. 3, the FD system is more susceptible to SI. In particular, the point where FD transmission is still more beneficial than HD transmission is now around $h_{\mathrm{LI}}=-120 \mathrm{~dB}$ and $h_{\mathrm{LI}}=$ $-126 \mathrm{~dB}$ instead of $h_{\mathrm{LI}}=-100 \mathrm{~dB}$. Figures $4 \mathrm{a}$ and c show that the spectral efficiency of the joint sum-power constraint case almost doubles as we move from $P_{2}=40 \mathrm{dBm}$ to $P_{2}=46 \mathrm{dBm}$. However, Figs. $4 \mathrm{~b}$ and $\mathrm{d}$ demonstrate that the throughput improvement is marginal for separate sum-power constraints. Here, since the increase in power budget only occurs to the relay, the UEs are less

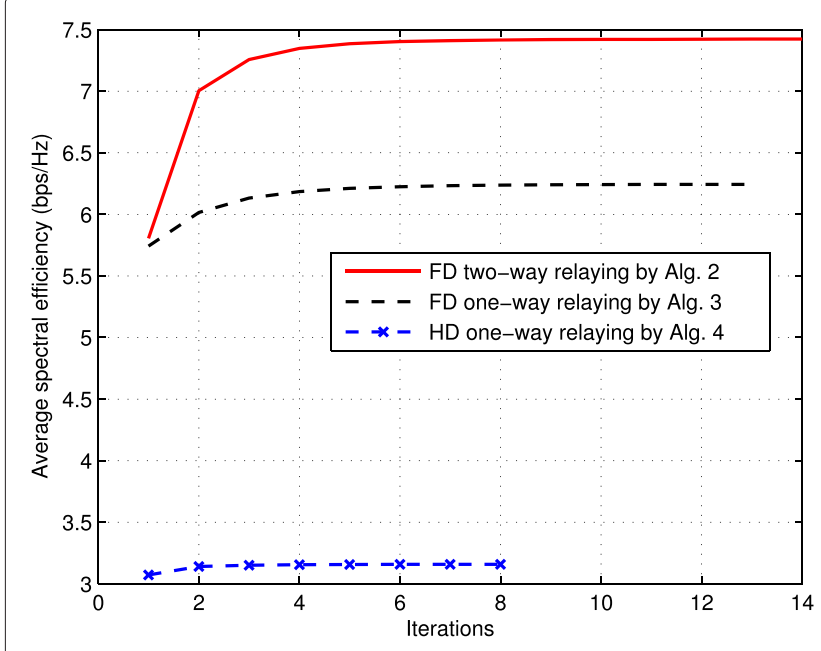

(a)

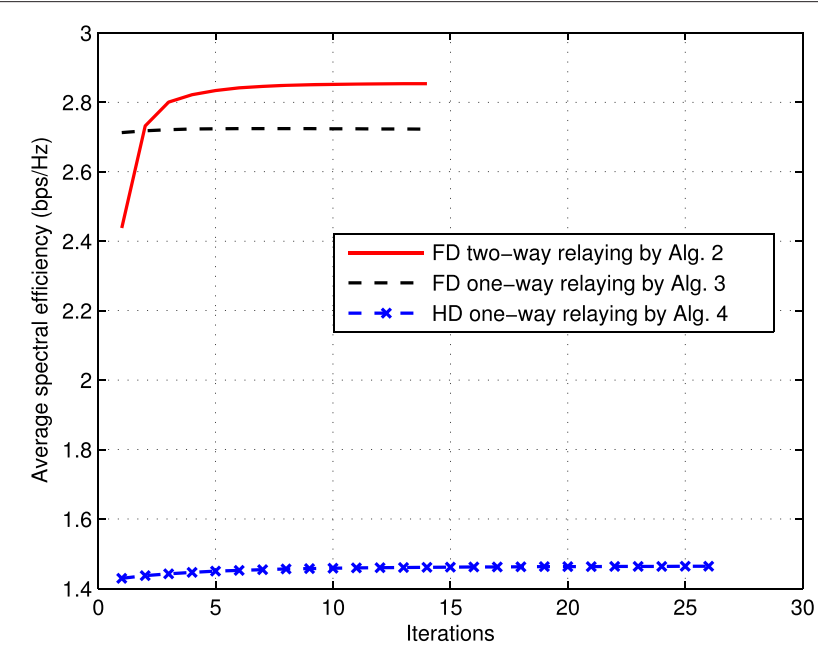

(b)

Fig. 5 Convergence for the UE-UE communication with BS relaying scenario with $P_{2}=40 \mathrm{dBm}$ and $h_{L I}=-140 \mathrm{~dB}$. a Joint sum-power constraint. b Separate sum-power constraint 
Table 1 Average number of iterations for $h_{\mathrm{LI}}=-140 \mathrm{~dB}$

\begin{tabular}{|c|c|c|c|c|c|c|}
\hline & \multicolumn{2}{|c|}{$U$ E relaying $\left(P_{2}=20 \mathrm{dBm}\right)$} & \multicolumn{2}{|c|}{$\mathrm{BS}$ relaying $\left(P_{2}=40 \mathrm{dBm}\right)$} & \multicolumn{2}{|c|}{$\mathrm{BS}$ relaying $\left(P_{2}=46 \mathrm{dBm}\right)$} \\
\hline & Joint power & Sep. power & Joint power & Sep. power & Joint power & Sep. power \\
\hline Alg. 2 & 14.26 & 14.78 & 17.56 & 16.45 & 11.81 & 20.76 \\
\hline Alg. 3 & 17.75 & 17.99 & 23.90 & 14.90 & 19.80 & 29.38 \\
\hline Alg. 4 & 14.49 & 15.86 & 20.57 & 21.14 & 17.56 & 23.75 \\
\hline
\end{tabular}

likely to exploit such an increase to enhance their power allocation.

Figure 5 illustrates the convergence of our proposed algorithms for a random channel realization in the scenario of UE-UE communication with BS relaying. Here we assume $P_{2}=40 \mathrm{dBm}$ and $h_{\mathrm{LI}}=-140 \mathrm{~dB}$. While convergence happens in at most 26 iterations, it can be as few as 8 iterations for a rather strict error tolerance $\epsilon=10^{-4}$. Table 1 details the number of iterations, averaged over 100 channel realizations, for the algorithms to converge in this case. Note that each iteration corresponds to evaluating a simple closed-form expression for the solution of a convex program, thus requiring a very little computational effort. Together with the small number of iterations, the total computational complexity is low even for our large-scale numerical examples with 4,096 channels.

In Figs. 3, 4 and 5, it is observed that the performance of the concerned algorithms in the joint sum-power constraint case is better than that in the separate sum-power constraint case. The reason is that the feasible set of the former problem contains that of the latter problem.
Finally, we compare our proposed algorithms with the two approaches of [16], namely, joint optimization with the high SNR assumption (for the joint sum-power constraint) and alternating optimization (for the separate sum-power constraints). Because the latter solutions only apply to the HD one-way relaying case, we compare their throughput performance with Algorithms 3 and 4. Here, we set $d_{1, R}=d_{R, 2}=1,000 \mathrm{~m}, \psi=20 \mathrm{~dB}$ and assume that each tap of the PDP follows the Rayleigh distribution. Figure 6a verifies that the high SNR approximation is not effective in the low-to-medium SNR scenario that we have simulated. This is demonstrated by the performance gap between the solution of [16] and our proposed solutions. Figure $6 \mathrm{~b}$ shows that Algorithms 3 and 4 perform as well as the alternating optimization of [16] for separate sum-power constraints. However, it should be recalled that the alternating optimization approach cannot be used for the joint sum-power constraint case. Also, the tailored solution in Algorithm 4 only applies to HD oneway relaying where SI does not exist, unlike the more general Algorithm 3 which applies even to FD one-way relaying.

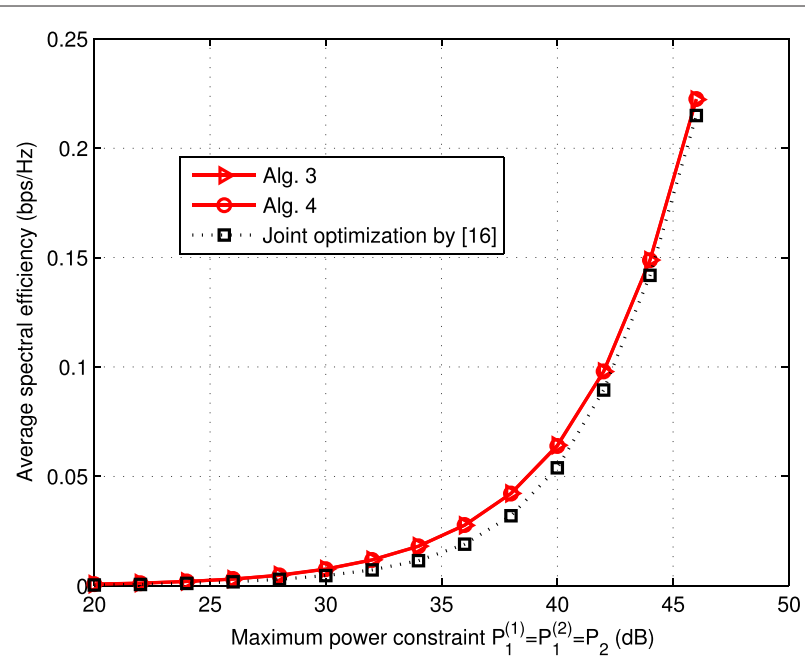

(a)

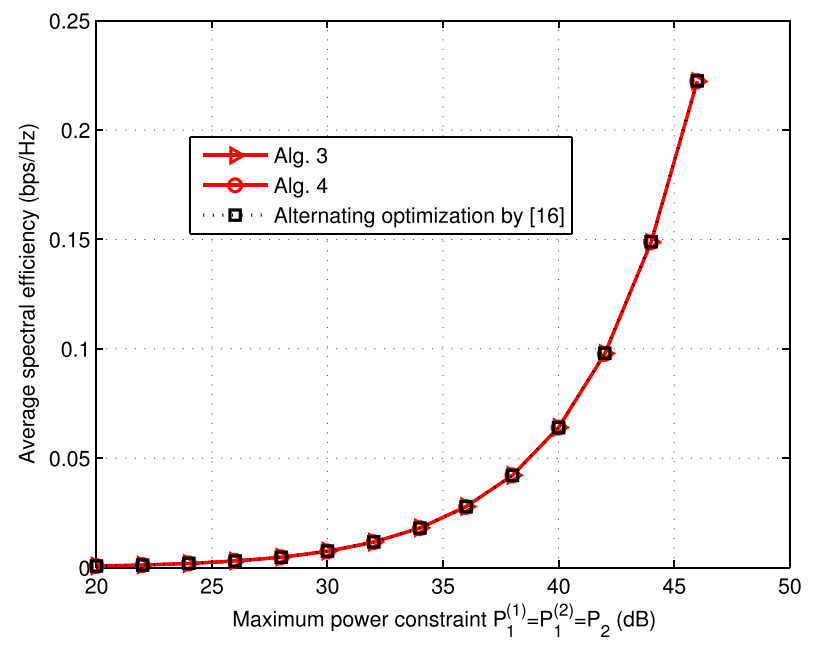

(b)

Fig. 6 Performance comparison for the HD one-way relaying case. a Joint sum-power constraint. b Separate sum-power constraints 


\section{Conclusions}

This paper addresses the problem of joint power allocation for a MIMO-OFDM network consisting of two FD users and one FD amplify-and-forward relay. The aim is to maximize the instantaneous total network throughput, subject to (i) the separate sum-power constraints at individual nodes or (ii) the joint sum-power constraint. To solve the highly nonconvex problem formulations, we have employed the successive convex approximation approach to develop novel iterative algorithms of extremely low complexity. A simple closed-form solution is available for the approximated convex program at each iteration. The proposed algorithms are shown to always converge to at least a local optimum. Our approach applies to the general case where any combination of one-way/two-way and HD/FD relaying is allowed. The advantages of our novel solutions have been confirmed by numerical examples.

\section{Endnotes}

${ }^{1}$ Noises are possibly amplified here. Moreover, $\mathcal{R}_{D, k}^{(\ell)}$ is no longer diagonal even when $\mathcal{R}_{D}^{(\ell)}$ is diagonal.

${ }^{2}$ As the noises in (7) are correlated, the subchannel-wise decoding is not optimal. Therefore, the objective function in (14) provides a lower bound instantaneous capacity of the network.

${ }^{3}$ One should use the obtained solution to compute the objective (14) in order to have the actual throughput performance.

\section{Appendix: Bisection Search}

The initial values $\lambda_{\mathrm{lo}}$ and $\lambda_{\mathrm{hi}}$ are given. For $\lambda:=\left(\lambda_{\mathrm{lo}}+\right.$ $\left.\lambda_{\text {hi }}\right) / 2$, use (41)-(42) to find $\left(x^{(1, \kappa+1)}, x^{(2, \kappa+1)}, y^{(\kappa+1)}\right)$. If $\sum_{i=1}^{M}\left(q_{i}^{(1)} \mathbf{x}_{i}^{(1)}+q_{i}^{(2)} \mathbf{x}_{i}^{(2)}+\mathbf{y}_{i}\right)>P$, set $\lambda_{\mathrm{lo}}:=\lambda$. If $\sum_{i=1}^{M}$ $\left(q_{i}^{(1)} \mathbf{x}_{i}^{(1)}+q_{i}^{(2)} \mathbf{x}_{i}^{(2)}+\mathbf{y}_{i}\right)<P$, set $\lambda_{\text {hi }}:=\lambda$. Proceed to the next bisection until $\sum_{i=1}^{M}\left(q_{i}^{(1)} \mathbf{x}_{i}^{(1)}+q_{i}^{(2)} \mathbf{x}_{i}^{(2)}+\mathbf{y}_{i}\right)=P$.

\section{Competing interests}

The authors declare that they have no competing interests.

\section{Author details}

${ }^{1}$ Centre for Health Technologies, University of Technology Sydney, Ultimo, NSW 2007, Australia. ${ }^{2}$ School of Electrical Engineering and Computing, The University of Newcastle, University Drive, Callaghan, NSW 2308, Australia.

Received: 29 June 2016 Accepted: 19 December 2016

Published online: 19 January 2017

\section{References}

1. DR Ng, WK Schober, Cross-Layer Scheduling for OFDMA Amplify-and-Forward Relay Networks. IEEE Trans. Veh. Technol. 59(3), 1443-1458 (2010)

2. YS Choi, H Shirani-Mehr, Simultaneous transmission and reception: algorithms, design and system level performance. IEEE Trans. Wireless Commun. 12(12), 5992-6010 (2013)
3. S Hong, J Brand, JI Choi, J Mehlman, S Katti, P Levis, Application of self-interference cancellation in $5 \mathrm{G}$ and beyond. IEEE Commun. Mag. 52(2), 114-21 (2014)

4. E Everett, A Sahai, A Sabharwal, Passive Self-Interference Suppression for Full-Duplex Infrastructure Nodes. IEEE Trans. Wireless Commun. 13(2), 680-694 (2014)

5. V Syrjala, M Valkama, L Anttila, T Riihonen, D Korpi, Analysis of oscillator phase-noise effects on self-interference cancellation in full-duplex OFDM radio trancseivers. IEEE Trans. Wireless Commun. 13(6), 2977-2990 (2014)

6. W Li, RD Murch, An investigation into baseband techniques for single-channel full duplex wireless communication systems. IEEE Trans. Wireless Commun. 13(9), 4794-4806 (2014)

7. B Rankov, A Wittneben, Spectral efficient protocols for half-duplex fading relay channels. IEEE J. Select Areas Commun. 25(2), 379-389 (2007)

8. K Jitvanichphaibool, R Zhang, YC Liang, Optimal Resource Allocation for Two-Way Relay-Assisted OFDMA. IEEE Trans. Veh. Technol. 58(7), 3311-3321 (2009)

9. HJ Yang, J Chun, A Paulraj, Asymptotic Capacity of the Separated MIMO Two-Way Relay Channel. IEEE Trans Inform. Theory. 57(11), 7542-7554 (2011)

10. X Yuan, T Yang, Collings IB.Multiple-Input Multiple-Output Two-Way Relaying: A Space-Division Approach. IEEE Trans Inform. Theory. 59(10), 6421-6440 (2013)

11. CK Ho, R Zhang, YC Liang, in Proc.IEEE Intl. Conf. Commun. (ICC). Two-Way Relaying over OFDM: Optimized Tone Permutation and Power Allocation, (Beijing, 2008), pp. 3908-3912

12. R Zhang, YC Liang, CC Chai, S Cui, Optimal beamforming for two-way multi-antenna relay channel with analogue network coding. IEEE J. Select Areas Commun. 27(5), 699-712 (2009)

13. M Zeng, R Zhang, S Cui, On design of collaborative beamforming for two-way relay networks. IEEE Trans Signal Process. 59(5), 2284-2295 (2011)

14. G Amarasuriya, C Tellambura, M Ardakani, Two-way amplify-and-forward multiple-input multiple-output relay networks with antenna selection. IEEE J. Select Areas Commun. 30(5), 1513-1529 (2012)

15. U Rashid, HD Tuan, HH Nguyen, Joint optimization of source precoding and relay beamforming in wireless $\mathrm{MIMO}$ relay networks. IEEE Trans. Commun. 62(2), 488-499 (2014)

16. I Hammerstrom, A Wittneben, Power allocation schemes for amplify-and-forward MIMO-OFDM relay links. IEEE Trans. Wireless Commun. 6(8), 2798-2802 (2007)

17. DWK Ng, ES Lo, R Schober, Dynamic resource allocation in MIMO-OFDMA systems with full-duplex and hybrid relaying. IEEE Trans Commun. 60(5), 1291-1303 (2012)

18. HH Kha, HD Tuan, HH Nguyen, Fast global optimal power allocation in wireless networks by local d.c. programming. IEEE Trans. Wireless Commun. 11, 511-515 (2012)

19. HH Kha, HD Tuan, HH Nguyen, Joint optimization of source power allocation and cooperative beamforming for SC-FDMA multi-user multi-relay networks. IEEE Trans Commun. 61(6), 2248-2259 (2013)

20. O Edfors, M Sandell, Berek van de JJ, SK Wilson, PO Borjesson, OFDM channel estimation by singular value decomposition. IEEE Trans Commun. 46(7), 931-939 (1998)

21. BP Day, AR Margetts, DW Bliss, P Schiniter, Full-duplex bidirectional MIMO: achievable rate under limited dynamic range. IEEE Trans. Signal Process. 60(7), 3702-3713 (2012)

22. BP Day, AR Margetts, DW Bliss, P Schiniter, Full-duplex MIMO relaying: achievable rate under limited dynamic range. IEEE J. Select Areas Commun. 30(8), 1541-1553 (2012)

23. M Ma, X Huang, B Hiao, YJ Guo, Optimal orthogonal precoding for power leakage suppression in DFT-based systems. IEEE Trans Commun. 59(3), 844-853 (2011)

24. A Ali, W Hamouda, Spectrum monitoring using energy ratio algorithm for OFDM-based cognitive radio networks. IEEE Trans. Wireless Commun. 4, 2257-2268 (2015)

25. J Abdoli, M Jia, J Ma, in Proc.IEEE Int. Workshop on Signal Process. Advances in Wireless Commun. (SPAWC). Filtered OFDM: a new waveform for future wireless systems, (Stockholm, 2015), pp. 66-70

26. M Duarte, A Sabharwal, V Aggarwal, R Jana, KK Ramakrishnan, CW Rice, et al, Design and characterization of a full-duplex multiantenna system for WiFi networks. IEEE Trans. Veh. Technol. 63(3), 1160-1177 (2014) 
27. CY Leow, Z Ding, KK Leung, Joint beamforming and power management for nonregenerative MIMO two-way relaying channels. IEEE Trans. Veh. Technol. 60(9), 4374-4383 (2011)

28. Tuy H, Convex Analysis and Global Optimization (second edition) (Springer, 2016)

29. J Papandriopoulos, JS Evans, SCALE: a low-complexity distributed protocal for spectrum balancing in multi-user DSL networks. IEEE Trans Inform. Theory. 55(8), 3711-3724 (2009)

30. BR Marks, GP Wright, A general inner approximation algorithm for nonconvex mathematical programs. Oper. Res. 26(4), 681-683 (1978)

31. European Telecommunications Standards Institute (ETSI). 3GPP TR 36.931 , version 9.0.0, Release 9 (2011)

32. YS Cho, J Kim, Yang WY, CG Kang, MIMO-OFDM Wireless Communications with MATLAB. (Wiley-IEEE Press, 2010)

33. A Goldsmith, Wireless Communications. (Cambridge University Press, 2004)

\section{Submit your manuscript to a SpringerOpen ${ }^{\odot}$ journal and benefit from:}

- Convenient online submission

- Rigorous peer review

- Immediate publication on acceptance

- Open access: articles freely available online

- High visibility within the field

- Retaining the copyright to your article

Submit your next manuscript at springeropen.com 